\title{
Determinacy of Wadge classes and subsystems of second order arithmetic
}

\author{
Takako Nemoto* \\ E-mail: sa4m20@math.tohoku.ac.jp \\ Mathematical Institute, Tohoku University, \\ Sendai, 980-8578, Japan
}

\begin{abstract}
In this paper we study the logical strength of the determinacy of infinite binary games in terms of second order arithmetic. We define new determinacy schemata inspired by the Wadge classes of Polish spaces and show the following equivalences over the system $\mathrm{RCA}_{0}^{*}$, which consists of the axioms of discrete ordered semi-ring with exponentiation, $\Delta_{1}^{0}$ comprehension and $\Pi_{0}^{0}$ induction, and which is known as a weaker system than the popular base theory $\mathrm{RCA}_{0}$ :
\end{abstract}

- $\operatorname{Bisep}\left(\Delta_{1}^{0}, \Sigma_{1}^{0}\right)-\operatorname{Det}^{*} \leftrightarrow \mathrm{WKL}_{0} ;$

- $\operatorname{Bisep}\left(\Delta_{1}^{0}, \Sigma_{2}^{0}\right)-$ Det $^{*} \leftrightarrow \operatorname{ATR}_{0}+\Sigma_{1}^{1}$ induction;

- $\operatorname{Bisep}\left(\Sigma_{1}^{0}, \Sigma_{2}^{0}\right)-$ Det $^{*} \leftrightarrow \operatorname{Sep}\left(\Sigma_{1}^{0}, \Sigma_{2}^{0}\right)$-Det* $\leftrightarrow \Pi_{1}^{1}-\mathrm{CA}_{0}$;

- $\operatorname{Bisep}\left(\Delta_{2}^{0}, \Sigma_{2}^{0}\right)$-Det ${ }^{*} \leftrightarrow \Pi_{1}^{1}-\mathrm{TR}_{0}$;

where Det* stands for the determinacy of infinite games in the Cantor space.

\section{Introduction}

We consider the following type of game: Two players, say player I and player II, alternatively choose an element of $X$ to form an infinite sequence $f$. Player I wins if and only if a given formula $\varphi(f)$ of $f$ holds. Player II wins if and only if I does not win. The formula $\varphi(f)$ is called a winning condition for player I. A determinacy statement asserts that one of the players has a winning strategy in such games. Within the framework of second order arithmetic, the strength of determinacy of games in the Baire space, i.e., the above games with $X=\mathbb{N}$, has been previously investigated in [3], [4], [8], [10], [11] and [12], and that of games in the Cantor space, i.e., the games with $X=2=\{0,1\}$, has been investigated in [5]. Their results are summarized in Table 1, where a subsystem of second order arithmetic and types of determinacy in the same line are pairwise equivalent over a suitable base theory ( $\mathrm{RCA}_{0}$; except for the last line), and where $\left(\Sigma_{2}^{0}\right)_{2}$ is the class $\Sigma_{2}^{0} \wedge \Pi_{2}^{0}$.

*Partly supported by the Grant-in-Aid for JSPS Fellows, The Ministry of Education, Culture, Sports, Science and Technology, Japan. 


\begin{tabular}{|c|c|c|}
\hline $\begin{array}{c}\text { Subsystem of } \\
\text { second order arithmetic }\end{array}$ & $\begin{array}{c}\text { Determinacy } \\
\text { in the Cantor space }\end{array}$ & $\begin{array}{c}\text { Determinacy } \\
\text { in the Baire space }\end{array}$ \\
\hline \hline WKL $L_{0}$ & $\Delta_{1}^{0}$ & \\
\hline $\mathrm{ACA}_{0}$ & $\Sigma_{1}^{0}$ & \\
\hline $\mathrm{ATR}_{0}$ & $\left.\Sigma_{1}^{0}\right)_{2}$ & \\
\hline$\Pi_{1}^{1}-\mathrm{CA}_{0}$ & $\Sigma_{2}^{0}$ & $\Delta_{1}^{0}$ \\
\hline$\Pi_{1}^{1}-\mathrm{TR}_{0}$ & & $\Sigma_{1}^{0}$ \\
\hline$\Sigma_{1}^{1}-\mathrm{ID}_{0}$ & & $\left(\Sigma_{1}^{0}\right)_{2}$ \\
\hline$\vdots$ & $\left(\Sigma_{2}^{0}\right)_{2}$ & $\Delta_{2}^{0}$ \\
\hline$\left[\Sigma_{1}^{1}\right]^{k}-\mathrm{ID}_{0}$ & $\vdots$ & $\Sigma_{2}^{0}$ \\
\hline$\vdots$ & $\left(\Sigma_{2}^{0}\right)_{k+1}$ & $\vdots$ \\
\hline$\left[\Sigma_{1}^{1}\right]^{\mathrm{TR}}-\mathrm{ID}_{0}$ & $\vdots$ & $\left(\Sigma_{2}^{0}\right)_{k}$ \\
\hline & $\Delta_{3}^{0}$ & $\vdots$ \\
\hline
\end{tabular}

Table 1: Results of earlier researches

In Table 1, we can find a large gap between $\Sigma_{2}^{0}$ and $\left(\Sigma_{2}^{0}\right)_{2}$ determinacy in the Cantor space. Thus, the aims of this paper are to find (1) a class whose determinacy in the Cantor space is equivalent to either $\Pi_{1}^{1}-\mathrm{CA}_{0}$ or $\Pi_{1}^{1}-\mathrm{TR}_{0}$ and (2) a finer hierarchy of determinacy in the Cantor space.

To find such a hierarchy, we consider the determinacy schemata which formalize, in the language of second order arithmetic, the determinacies whose winning conditions are in Borel Wadge classes in Polish spaces and also investigate the strengths of the schemata. Borel Wadge classes, which are defined as those classes of Borel sets that are closed under continuous pre-images, are known to form a finer hierarchy than the Hausdorff difference hierarchy (cf. [2], [13]). Since continuous pre-images preserve Boolean operations, this hierarchy seems to be the finest among those hierarchies to which we can define corresponding determinacy schemata at least in an obvious manner.

If we continue to work over RCA $A_{0}$, we will find that weak König's lemma and $\operatorname{Bisep}\left(\Delta_{1}^{0}, \Sigma_{1}^{0}\right), \Sigma_{1}^{0}$ and $\Delta_{1}^{0}$ determinacies in the Cantor space are pairwise equivalent over $\operatorname{RCA}_{0}$, where $\operatorname{Bisep}\left(\Delta_{1}^{0}, \Sigma_{1}^{0}\right)$ determinacy formalizes the determinacy of the Wadge class immediately above the class $\boldsymbol{\Sigma}_{1}^{0}$ of open sets (see Figure 2 and [2]). However, the proof of $\operatorname{Bisep}\left(\Delta_{1}^{0}, \Sigma_{1}^{0}\right)$ determinacy essentially needs $\Sigma_{1}^{0}$ induction, while that of $\Sigma_{1}^{0}$ and $\Delta_{1}^{0}$ determinacies does not (in [5]). To shed light on the differences among the strengths of the determinacies of those classes, we replace the base theory with RCA which lacks $\Sigma_{1}^{0}$ induction. Although almost all the results in Table 1 were equivalences over $R C A_{0}$, it is routine to check that the base theory can be replaced with $\mathrm{RCA}_{0}^{*}$.

This paper proves the following theorem, and as a result, Table 1 is enhanced into Table 2.

Theorem RCA $A_{0}^{*}$ proves the following four equivalences, where Det* (resp. Det) represents the determinacy of infinite games in the Cantor space (resp. the Baire space).

- $\operatorname{Bisep}\left(\Delta_{1}^{0}, \Sigma_{1}^{0}\right)$-Det ${ }^{*} \leftrightarrow \mathrm{WKL}_{0}$

- $\operatorname{Bisep}\left(\Delta_{1}^{0}, \Sigma_{2}^{0}\right)-$ Det $^{*} \leftrightarrow \operatorname{ATR}_{0}+\Sigma_{1}^{1}$ induction

- $\operatorname{Bisep}\left(\Sigma_{1}^{0}, \Sigma_{2}^{0}\right)-\operatorname{Det}^{*} \leftrightarrow \operatorname{Sep}\left(\Sigma_{1}^{0}, \Sigma_{2}^{0}\right)-\operatorname{Det}^{*} \leftrightarrow \operatorname{Bisep}\left(\Delta_{1}^{0}, \Sigma_{1}^{0}\right)$-Det $\leftrightarrow \Pi_{1}^{1}-\mathrm{CA}_{0}$.

- $\operatorname{Bisep}\left(\Delta_{2}^{0}, \Sigma_{2}^{0}\right)$-Det ${ }^{*} \leftrightarrow \Pi_{1}^{1}-\mathrm{TR}_{0}$. 


\begin{tabular}{|c|c|c|}
\hline $\begin{array}{c}\text { Subsystem of } \\
\text { second order arithmetic }\end{array}$ & $\begin{array}{c}\text { Determinacy } \\
\text { in the Cantor space }\end{array}$ & $\begin{array}{c}\text { Determinacy } \\
\text { in the Baire space }\end{array}$ \\
\hline \hline WKL & $\begin{array}{c}\Delta_{1}^{0} \\
\Sigma_{1}^{0}\end{array}$ & - \\
\hline $\mathrm{WKL}_{0}$ & $\operatorname{Bisep}\left(\Delta_{1}^{0}, \Sigma_{1}^{0}\right)$ & - \\
\hline $\mathrm{ACA}_{0}$ & $\left(\Sigma_{1}^{0}\right)_{2}$ & - \\
\hline $\mathrm{ATR}_{0}$ & $\Delta_{2}^{0}$ & $\Delta_{1}^{0}$ \\
& $\Sigma_{2}^{0}$ & $\Sigma_{1}^{0}$ \\
\hline $\mathrm{ATR}_{0}+\Sigma_{1}^{1}$ induction & $\operatorname{Bisep}\left(\Delta_{1}^{0}, \Sigma_{2}^{0}\right)$ & $?$ \\
\hline & $\operatorname{Bisep}\left(\Sigma_{1}^{0}, \Sigma_{2}^{0}\right)$ & $\operatorname{Bisep}\left(\Delta_{1}^{0}, \Sigma_{1}^{0}\right)$ \\
$\Pi_{1}^{1}-\mathrm{CA}_{0}$ & $\vdots$ & $\left(\Sigma_{1}^{0}\right)_{2}$ \\
& $\operatorname{Sep}\left(\Sigma_{1}^{0}, \Sigma_{2}^{0}\right)$ & $\Delta_{2}^{0}$ \\
\hline$\Pi_{1}^{1}-\mathrm{TR}_{0}$ & $\operatorname{Bisep}\left(\Delta_{2}^{0}, \Sigma_{2}^{0}\right)$ & $\Sigma_{2}^{0}$ \\
\hline$\Sigma_{1}^{1}-\mathrm{ID}_{0}$ & $\left(\Sigma_{2}^{0}\right)_{2}$ & $\vdots$ \\
\hline$\vdots$ & $\vdots$ & $\left(\Sigma_{2}^{0}\right)_{k}$ \\
\hline$\left[\Sigma_{1}^{1}\right]^{k}-\mathrm{ID}_{0}$ & $\left(\Sigma_{2}^{0}\right)_{k+1}$ & $\vdots$ \\
\hline$\vdots$ & $\vdots$ & $\Delta_{3}^{0}$ \\
\hline$\left[\Sigma_{1}^{1}\right]^{\mathrm{TR}}-\mathrm{ID}_{0}$ & $\Delta_{3}^{0}$ & \\
\hline & & \\
\hline & & \\
\hline
\end{tabular}

Table 2: Results of the present study

Table 2 indicates that a subsystem of second order arithmetic and types of determinacy in the same line are pairwise equivalent over $\mathrm{RCA}_{0}^{*}$ (except for the last line).

The proof of each equivalence is as follows. When we prove $\Gamma$ determinacy in some system Sys, we reduce a $\Gamma$ game $\varphi(f)$ to an "easier" one $\varphi^{*}(f)$ such that the determinacy of $\varphi^{*}(f)$ can be proved in Sys. The key point in this direction is how to reduce the game within a restricted comprehension axiom. Conversely, we prove that $\Gamma$ determinacy implies the set comprehension axiom that characterizes Sys as follows. For any formula $\varphi(k)$ in a given class for which comprehension axiom characterizes the system Sys, we construct the following game whose determinacy is implied by $\Gamma$ determinacy:

- Player I chooses $k$ and asks whether $\varphi(k)$.

- Player II answers yes or no.

- Player II wins if and only if her answer is correct.

In such a game, player I cannot win if player II answers correctly. By $\Gamma$ determinacy, player II has a winning strategy, which provides the desired set $\{n: \varphi(n)\}$.

The outline of this paper is as follows. Subsection 2.1 introduces basic definitions and notations. Subsection 2.2 is a short survey of fundamental results of the new base theory RCA $A_{0}^{*}$ and related systems. Subsection 2.3 formulates determinacy in second order arithmetic and overviews some basic properties. Subsection 2.4 overviews previously known results of Wadge classes in descriptive set theory. Inspired by this, Subsection 2.5 defines new determinacy schemata, e.g., $\operatorname{Bisep}\left(\Delta_{1}^{0}, \Sigma_{1}^{0}\right)$ determinacy, which play important roles in this paper. Section 3 proves the equivalence over RCA* between $\mathrm{WKL}_{0}$ and $\operatorname{Bisep}\left(\Delta_{1}^{0}, \Sigma_{1}^{0}\right)$ determinacy in the Cantor space. This result shows that, to 
investigate the strengths of determinacies beyond $\operatorname{Bisep}\left(\Delta_{1}^{0}, \Sigma_{1}^{0}\right)$ determinacy, it is irrelevant whether the base theory contains $\Sigma_{1}^{0}$ induction. The following sections find several determinacies in the Cantor space that are equivalent to $\mathrm{ATR}_{0}+\Sigma_{1}^{1}$ induction, $\Pi_{1}^{1}-\mathrm{CA}_{0}$, and $\Pi_{1}^{1}-\mathrm{TR}_{0}$.

\section{Preliminaries}

In this section, we recall some basic definitions and facts about second order arithmetic and about determinacy. We also define various determinacy schemata.

\subsection{Basic definitions and notations in second order arithmetic}

The language $\mathrm{L}_{1}$ of first order arithmetic consists of $+, \cdot, 0,1,=,<$, number variables $x, y, \ldots$, propositional connectives and number quantifiers. The language $\mathrm{L}_{2}$ of second order arithmetic consists of $\mathrm{L}_{1}$ plus set variables $X, Y, \ldots$, set quantifiers and $\in$. Terms and formulae are defined in the usual way. A formula is $\Pi_{0}^{0}, \Sigma_{0}^{0}$ or $\Delta_{0}^{0}$ if it is built up from atomic formulae by propositional connectives and bounded number quantifiers $\forall x<t$ and $\exists x<t$. A $\Sigma_{n}^{0}$ (resp. $\Pi_{n}^{0}$ ) formula is one consisting of $n$ number quantifiers beginning with an existential (resp. universal) one followed by a $\Pi_{0}^{0}$ formula. A formula is $\Sigma_{0}^{1}$ or $\Pi_{0}^{1}$ if it does not contain set quantifiers. A $\Sigma_{n}^{1}$ (resp. $\Pi_{n}^{1}$ ) formula is one consisting of $n$ set quantifier beginning with an existential (resp. universal) one followed by a $\Pi_{0}^{1}$ formula.

In what follows, $\Gamma$ is a class of formulae, e.g., $\Sigma_{n}^{i}$ and $\Pi_{n}^{i}$.

Definition 2.1 We consider the following schemata.

$\Gamma$ comprehension: $\exists Y \forall x(x \in Y \leftrightarrow \varphi(x))$, where $\varphi(x)$ is a $\Gamma$ formula in which $Y$ does not occur freely.

Bounded $\Gamma$ comprehension: $\forall n \exists Y \forall i(i \in Y \leftrightarrow(i<n \wedge \varphi(n)))$, where $\varphi(x)$ is a $\Gamma$ formula in which $Y$ does not occur freely.

$\Gamma$ induction: $(\varphi(0) \wedge \forall n(\varphi(n) \rightarrow \varphi(n+1))) \rightarrow \forall n \varphi(n)$, where $\varphi(x)$ is a $\Gamma$ formula.

$\Gamma$ transfinite induction: $\operatorname{WO}\left(Z,<_{Z}\right) \wedge \forall j\left(\forall i\left(i<_{Z} j \rightarrow \varphi(i)\right) \rightarrow \varphi(j)\right) \rightarrow \forall j \varphi(j)$, where $\varphi(x)$ is a $\Gamma$ formula and where $\operatorname{WO}\left(Z,<_{Z}\right)$ is the $\Pi_{1}^{1}$ formula which asserts that $\left(Z,<_{Z}\right)$ is well-ordered, i.e., $\left(Z,<_{Z}\right)$ is a linear ordering without infinite $<_{Z}$-descending chains.

Definition 2.2 $\left(R C A_{0}\right) R_{C A}$ is the formal system in the language $L_{2}$ which consists of the axioms of discrete ordered semi-ring for $(\mathbb{N},+, \cdot, 0,1,<)$ plus the schemata of $\Sigma_{1}^{0}$ induction and of $\Delta_{1}^{0}$ comprehension:

$$
\forall n(\varphi(n) \leftrightarrow \psi(n)) \rightarrow \exists Y \forall n(\varphi(n) \leftrightarrow n \in Y),
$$

where $\varphi(x)$ is a $\Sigma_{1}^{0}$ formula without free occurrences of $Y$, and where $\psi(x)$ is a $\Pi_{1}^{0}$ formula.

Over $\mathrm{RCA}_{0}, \Gamma$ comprehension implies not only $\Gamma$ induction but also $\Gamma$ transfinite induction.

Lemma 2.3 Over $\mathrm{RCA}_{0}$, $\Gamma$ comprehension implies $\Gamma$ transfinite induction.

Proof. Let $\left(Y, \prec_{Y}\right)$ be a well-ordering and $\varphi(x)$ a $\Gamma$ formula. Assume $\forall j\left(\forall i\left(i \prec \prec_{Y} \rightarrow \varphi(i)\right) \rightarrow\right.$ $\varphi(j))$ and $\exists k \neg \varphi(k)$. By $\Gamma$ comprehension and $\Delta_{1}^{0}$ comprehension, we have the set $Z=\{k: \neg \varphi(k)\}$. Since $Z$ is non-empty, we can take $k \in Z$. Note that if $l \in Z$, then $m \prec_{Y} l$ for some $m \in Z$ by the assumption. Define $f: \mathbb{N} \rightarrow Z$ by (unbounded) primitive recursion as follows:

$$
f(0)=k \quad \text { and } \quad f(n+1)=\text { the }<\text {-minimum } l \in Z \text { such that } l \prec_{Y} f(n) .
$$

Then $f(n+1) \prec_{Y} f(n)$ holds for all $n$, which contradicts the well-orderedness of $\left(Y, \prec_{Y}\right)$.

As mentioned in Introduction, we need a weaker base theory $\mathrm{RCA}_{0}^{*}$. 
Definition $2.4\left(\mathrm{RCA}_{0}^{*}\right)$ Let $\mathrm{L}_{i}(\exp )(i=1,2)$ be $\mathrm{L}_{i}$ augmented by a binary operation symbol $\exp (m, n)$, which is intended to denote exponentiation. For each $n<\omega, \Sigma_{n}^{i}$ and $\Pi_{n}^{i}$ formulae of $\mathrm{L}_{2}(\exp )$ are defined accordingly. $\mathrm{RCA}_{0}^{*}$ is the $\mathrm{L}_{2}(\exp )$-theory consisting of the axiom of discrete ordered semi-ring for $(\mathbb{N},+, \cdot, 0,1,<)$ plus exponentiation axioms, $m^{0}=1, m^{n+1}=m^{n} \cdot m$, where $t_{1}^{t_{2}}$ is an abbreviation for $\exp \left(t_{1}, t_{2}\right)$, and the schemata of $\Delta_{1}^{0}$ comprehension and of $\Pi_{0}^{0}$ induction.

Note that the $\mathrm{L}_{2}$-theory $\mathrm{RCA} \mathrm{A}_{0}$ can be regarded as the $\mathrm{L}_{2}(\exp )$-theory $\mathrm{RCA} \mathrm{A}_{0}^{*}$ plus $\Sigma_{1}^{0}$ induction, since exp can be defined in $\mathrm{RCA}_{0}$.

Notation 2.5 (sequences) The set of all infinite sequences from $X$ (i.e., functions from $\mathbb{N}$ to $X$ ) is denoted by $X^{\mathbb{N}}$. We call $2^{\mathbb{N}}=\{0,1\}^{\mathbb{N}}$ the Cantor space and $\mathbb{N}^{\mathbb{N}}$ the Baire space. The set of all finite sequences from $X$ is denoted by $X^{<\mathbb{N}}$. Note that the empty sequence, denoted by \langle\rangle , belongs to $X^{<\mathbb{N}}$.

Fix any $s, t \in X^{\mathbb{N}} .|s|$ denotes the length of $s$ and $s(i)$ denotes the $(i+1)$-th element of $s$ for $i<|s|$. The concatenation of $s$ and $t$, denoted by $s * t$, is $\langle s(0), s(1), \ldots, s(|s|-1), t(0), t(1), \ldots, t(|t|-1)\rangle$. If $f \in X^{\mathbb{N}}, s * f$ denotes $\langle s(0), s(1), \ldots, s(|s|-1), f(0), f(1), \ldots, f(n), \ldots\rangle$. For $s \in X^{<\mathbb{N}}$ and $n \leq|s|$, $s[n]$ is the $n$-th initial segment of $s$, i.e., $\langle s(0), \ldots, s(n-1)\rangle$. If $f$ is an infinite sequence, $f[n]$ denotes $\langle f(0), \ldots, f(n-1)\rangle$. If $s=t[k]$ for some $k \leq|t|, s$ is called an initial segment of $t, s \subseteq t$ for notation. If $n \leq|s|, s \ominus n$ is the sequence with the first $n$ elements removed from $s$, i.e., $\langle s(n), s(n+1), \ldots, s(|s|-1)\rangle$. If $f$ is in $X^{\mathbb{N}}, f \ominus n$ is $g$ defined by $g(k)=f(n+k)$ for $k \in \mathbb{N}$.

Note that, since a sequence $s$ of $X^{<\mathbb{N}}$ can be coded by a natural number in $\mathrm{RCA}_{0}^{*}$, the relations $s \in X^{<\mathbb{N}}, s=\langle\rangle,|s|=n, s(i)=n, s \subseteq t, s=t * u, s=t \ominus n, s=t[n]$, etc., are formally defined as $\Pi_{0}^{0}$ formulae in $\mathrm{RCA}_{0}^{*}$. See [8, Sections II.1-2] for more details. (Although they work in $\mathrm{RCA}_{0}$ in $[8$, Sections II.1-2], the base theory can be replaced by RCA $A_{0}^{*}$.) By coding of finite sequences by natural numbers, we can define transfinite recursion and axioms of choice.

Definition 2.6 We define the following schemata.

$\Gamma$ transfinite recursion: $\mathrm{WO}\left(Y, \prec_{Y}\right) \rightarrow \exists Z \forall j \forall k\left(\langle k, j\rangle \in Z \leftrightarrow \theta\left(k,(Z)^{j}\right)\right)$, where $\theta(x, Y)$ is a $\Gamma$ formula in which $Z$ does not occur freely, and where $(Z)^{j}=\left\{\langle m, i\rangle \in Z: i \prec_{Y} j\right\}$.

$\Gamma$ axiom of choice: $\forall n \exists Y \varphi(n, Y) \rightarrow \exists Z \forall n \varphi\left(n,(Z)_{n}\right)$, where $\varphi(x, Y)$ is a $\Gamma$ formula $\varphi$ in which $Z$ does not occur freely, and where $(Z)_{n}=\{m:\langle m, n\rangle \in Z\}$.

\subsection{The systems $\mathrm{RCA}_{0}^{*}$ and $\mathrm{WKL}_{0}^{*}$}

In the last subsection, we defined $\mathrm{RCA}_{0}^{*}$. Here we see how $\mathrm{RCA}_{0}^{*}$ differs from $\mathrm{RCA}$. We also define $\mathrm{WKL}_{0}$ and $\mathrm{WKL}_{0}^{*}$ by adding weak König's lemma, and investigate the relation among them.

First we overview the provable definability of functions in $\mathrm{RCA}_{0}^{*}$. By $\Delta_{1}^{0}$ comprehension, $\mathrm{RCA}_{0}^{*}$ proves the existence of the constant function $g_{0}(n)=0$, the successor function $g_{\mathrm{suc}}(n)=n+1$, the addition $g_{+}(n, m)=n+m$, the multiplication $g \cdot(n, m)=n \cdot m$, the exponentiation $g_{\exp }(n, m)=n^{m}$ and the projection functions $p_{i}^{n}\left(\left\langle k_{1}, \ldots, k_{i}, \ldots, k_{n}\right\rangle\right)=k_{i}$. The universe of provably definable functions of $\mathrm{RCA}_{0}^{*}$ is closed under the operations of Lemma 2.7 below. Therefore every elementary function can be defined in $\mathrm{RCA}_{0}^{*}$ (cf. [9, Section 4]).

Lemma 2.7 ([9, 2.1. Lemma and 2.2. Lemma $])$ The following are provable in $\mathrm{RCA}_{0}^{*}$.

Composition If $g_{0}: Y \rightarrow Z$ and $g_{1}: Z \rightarrow W$, there is $h: Y \rightarrow W$ defined by $h(i)=g_{1}\left(g_{0}(i)\right)$.

Bounded primitive recursion If $g_{0}: \mathbb{N}^{k} \rightarrow \mathbb{N}, g_{1}: \mathbb{N}^{k+2} \rightarrow \mathbb{N}$ and $g_{2}: \mathbb{N}^{k+1} \rightarrow \mathbb{N}$, there exists the unique $h: \mathbb{N}^{k+1} \rightarrow \mathbb{N}$ defined by

$$
\begin{aligned}
h\left(0, n_{1}, \ldots, n_{k}\right) & =g_{0}\left(n_{1}, \ldots, n_{k}\right), \\
h\left(m+1, n_{1}, \ldots, n_{k}\right) & =\min \left\{g_{1}\left(m, h\left(m, n_{1}, \ldots, n_{k}\right), n_{1}, \ldots, n_{k}\right), g_{2}\left(m, n_{1}, \ldots, n_{k}\right)\right\} .
\end{aligned}
$$


Minimization If $g: \mathbb{N}^{k+1} \rightarrow \mathbb{N}$ and if all $\left\langle n_{1}, \ldots, n_{k}\right\rangle \in \mathbb{N}^{k}$ have $m \in \mathbb{N}$ with $g\left(m, n_{1}, \ldots, n_{k}\right)=1$, there is $h: \mathbb{N}^{k} \rightarrow \mathbb{N}$ defined by $h\left(n_{1}, \ldots, n_{k}\right)=$ the least $m$ with $g\left(m, n_{1}, \ldots, n_{k}\right)=1$.

The following lemma shows that the first order part of $\mathrm{RCA}_{0}^{*}$ contains $\Sigma_{1}^{0}$ collection.

Lemma $2.8\left(\Sigma_{1}^{0}\right.$ collection, $\left[9,4.1\right.$. Lemma]) RCA proves $\Sigma_{1}^{0}$ collection, i.e.,

$$
\forall n \exists m \varphi(n, m) \rightarrow \forall n \exists m \forall k<n \exists l<m \varphi(k, l), \quad \text { for any } \Sigma_{1}^{0} \text { formula } \varphi(x, y) .
$$

Second, we consider weak König's lemma and add this to our systems.

A set $T \subseteq X^{<\mathbb{N}}$ is a tree over $X$ if it is closed under initial segments, i.e., $t \in T$ and $s \subseteq t$ imply $s \in T$. A tree is infinite if, for any $n$, there exists $s \in T$ with $|s|=n . f: \mathbb{N} \rightarrow X$ is a path through a tree $T$ if each initial segment $f[m]$ of $f$ is in $T$.

Definition 2.9 Weak König's lemma asserts that every infinite tree over $\{0,1\}$ has a path. $\mathrm{WKL}_{0}$ and $\mathrm{WKL}_{0}^{*}$ are the systems $\mathrm{RCA}_{0}$ and $\mathrm{RCA}_{0}^{*}$, respectively, plus weak König's lemma.

Clearly $\mathrm{WKL}_{0}$ includes $\mathrm{RCA}_{0}$. Actually, this inclusion is strict (see [8, Section VIII. 2]). This implies that $\mathrm{WKL}_{0}^{*}$ also strictly includes $\mathrm{RCA}_{0}^{*}$.

Informally, we have the following equivalences. (See [8, Section X.4])

$$
\mathrm{RCA}_{0} \equiv \mathrm{RCA}_{0}^{*}+\Sigma_{1}^{0} \text { induction, and } \mathrm{WKL}_{0} \equiv \mathrm{WKL}_{0}^{*}+\Sigma_{1}^{0} \text { induction. }
$$

Let us consider the first order part and the consistency strength of these systems.

$\mathrm{I} \Sigma_{1}$ is the $\mathrm{L}_{1}$ theory consisting of the axioms of discrete ordered semi-ring for $(\mathbb{N},+, \cdot, 0,1,<)$ and of $\Sigma_{1}$ induction. $\mathrm{I} \Delta_{0}(\exp )$ is the $\mathrm{L}_{1}(\exp )$ theory consisting of the axioms of discrete ordered semi-ring with exponentiation for $(\mathbb{N},+, \cdot, \exp , 0,1,<)$ and of $\Delta_{0}$ induction. $\mathrm{B} \Sigma_{1}(\exp )$ is the system $\mathrm{I} \Delta_{0}(\exp )$ plus $\Sigma_{1}$ collection. By the same proof as in [6] we can see that $\mathrm{I} \Delta_{0}(\exp )$ is strictly included in $\mathrm{B} \Sigma_{1}(\exp )$ although the original proof in [6] is for the language $\mathrm{L}_{1}$.

Fact 2.10 (conservation theorems, [8, Sections IX.1-IX.3] and [9]) The first order part of RCA and of $\mathrm{WKL}_{0}$ is $\mathrm{I}_{1}$. WKL $\mathrm{W}_{0}$ is conservative over $\mathrm{RCA}_{0}$ for $\Pi_{1}^{1}$ sentences, i.e., for any $\Pi_{1}^{1}$ sentence $\varphi, \mathrm{WKL}_{0} \vdash \varphi$ if and only if $\mathrm{RCA}_{0} \vdash \varphi$. $\mathrm{WKL}_{0}, \mathrm{RCA}_{0}$ and $\mathrm{I}_{1}$ have the same consistency strength as PRA (See [8, Section IX. 3] for its definition and basic properties.) and are conservative over PRA for $\Pi_{2}^{0}$ sentences.

Analogously, the first order part of $\mathrm{RCA}_{0}^{*}$ and of $\mathrm{WKL}_{0}^{*}$ is $\mathrm{B} \Sigma_{1}(\exp )$. $\mathrm{WKL}_{0}^{*}$ is conservative over $\mathrm{RCA}_{0}^{*}$ for $\Pi_{1}^{1}$ sentences. $\mathrm{WKL}_{0}^{*}, \mathrm{RCA}_{0}^{*}$ and $\mathrm{B} \Sigma_{1}(\exp )$ have the same consistency strength as $\mathrm{I} \Delta_{0}(\exp )$ and are conservative over $\mathrm{I} \Delta_{0}(\exp )$ for $\Pi_{2}^{0}$ sentences.

It is shown in [8, Theorem II.8.11] that $\mathrm{RCA}_{0}$ proves the consistency of $\mathrm{I} \Delta_{0}(\exp )$, and so does $\mathrm{I} \Sigma_{1}$. On the other hand, I $\Delta_{0}(\exp )$ cannot prove the consistency of itself and so neither can $\mathrm{B} \Sigma_{1}(\exp )$, since the consistency statement is $\Pi_{1}^{0}$. Therefore $\mathrm{I} \Sigma_{1}$ strictly includes $\mathrm{B} \Sigma_{1}(\exp )$, and the implications from $\mathrm{RCA}_{0}$ to $\mathrm{RCA}_{0}^{*}$ and from $\mathrm{WKL}_{0}$ to $\mathrm{WKL}_{0}^{*}$ are also strict. Moreover the consistency strength of $\mathrm{WKL}_{0}^{*}, \mathrm{RCA}_{0}^{*}, \mathrm{~B} \Sigma_{1}$ and $\mathrm{I} \Delta_{0}(\exp )$ is strictly weaker than that of $\mathrm{WKL}_{0}, \mathrm{RCA}_{0}, \mathrm{I} \Sigma_{1}$ and PRA.

The relations among these weak systems are described in Figure 1, where usual (one-head) arrows indicate strict implication with conservation for indicated classes of sentences, where two-head arrows indicate strict implication with different consistency strength and where lines without heads indicate full conservation between systems in $\mathrm{L}_{1}(\exp )$ and their counterparts in $\mathrm{L}_{2}(\exp )$ (e.g., for any $\mathrm{L}_{1}(\exp )$ sentence $\varphi, \mathrm{WKL}_{0}^{*} \vdash \varphi$ if and only if $\left.\mathrm{B} \Sigma_{1}(\exp ) \vdash \varphi\right)$. 


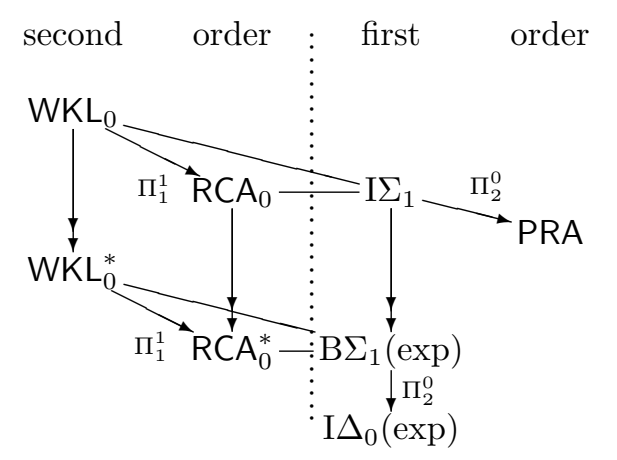

Figure 1: Relations among weak systems of arithmetic

\subsection{Determinacy in second order arithmetic}

For a given formula $\varphi(f)$ with a distinguished function variable $f \in X^{\mathbb{N}}$, a game $\varphi(f)$ in $X^{\mathbb{N}}$ is defined as follows: Two players, say player I and player II, alternately choose an element $x$ in $X$ to form $f \in X^{\mathbb{N}}$ which is called the resulting play. Player I wins if and only if $\varphi(f)$ holds. Player II wins if and only if player I does not win. In this paper, we assume that player I is male and that player II is female.

We regard a class $\Gamma$ of formulae with a distinguished function variable as a class of games.

Remark 2.11 $\mathrm{L}_{2}$ does not formally have function variables. Since the assertion " $G$ is the graph of a function $f: \mathbb{N} \rightarrow \mathbb{N}$ " is $\Pi_{2}^{0}$, this point matters in a system without arithmetical $\left(\Pi_{0}^{1}\right)$ comprehension. However, we treat only the case where $X=\{0,1\}$ in such systems, and so we can regard $f$ as a set variable by identifying $f(n)=0$ and $f(n)=1$ with $n \notin f$ and $n \in f$ respectively. Therefore we do not care about this difference.

Notation 2.12 (strategy) For a game in $X^{\mathbb{N}}$, a strategy $\sigma$ for player I (resp. II) is a function assigning an element of $X$ to each finite sequence from $X$ of even (resp. odd)-length. $\mathcal{S}_{\mathrm{I}}^{X}$ (resp. $\mathcal{S}_{\text {II }}^{X}$ ) is the set of all the strategies for player I (resp. II) in a game in $X^{\mathbb{N}}$. Note that $\mathcal{S}_{\mathrm{I}}^{X}$ and $\mathcal{S}_{\text {II }}^{X}$ can be regarded as $\mathbb{N}^{\mathbb{N}}$ if $X=\mathbb{N}$ and $2^{\mathbb{N}}$ if $X=\{0,1\}$ in $\mathrm{RCA}_{0}^{*}$, by a suitable coding of finite sequences. If players I and II follow strategies $\sigma$ and $\tau$ respectively, the resulting play is uniquely determined and denoted by $\sigma \otimes \tau$.

For any strategy $\tau$ for player II, $k^{\tau}$ is the finite play of length $2 k$ in which player I plays 0 at all of his turns and in which player II plays following $\tau$. For example, $2^{\tau}$ is the sequence $\langle 0, \tau(\langle 0\rangle), 0, \tau(\langle 0, \tau(\langle 0\rangle), 0\rangle)\rangle$.

A strategy $\sigma$ for a player is a winning strategy if the player wins $\varphi(f)$ as long as he or she plays following it. The assertion that $\sigma$ is a winning strategy for player I (resp. II) in game $\varphi(f)$ in $X^{\mathbb{N}}$ can be written $\forall \tau \in \mathcal{S}_{\mathrm{II}}^{X} \varphi(\sigma \otimes \tau)$ (resp. $\forall \tau \in \mathcal{S}_{\mathrm{I}}^{X} \neg \varphi(\tau \otimes \sigma)$ ). A game $\varphi(f)$ is determinate if one of the players has a winning strategy. For a game $\varphi(f)$ in $X^{\mathbb{N}}$, we use the following abbreviation:

$$
\operatorname{Det}^{X}[\varphi] \equiv \exists \sigma \in \mathcal{S}_{\mathrm{I}}^{X} \forall \tau \in \mathcal{S}_{\mathrm{II}}^{X} \varphi(\sigma \otimes \tau) \vee \exists \tau \in \mathcal{S}_{\mathrm{II}}^{X} \forall \sigma \in \mathcal{S}_{\mathrm{I}}^{X} \neg \varphi(\sigma \otimes \tau),
$$

which asserts that $\varphi(f)$ is determinate. The following schema of $\Gamma$ determinacy asserts that all the $\Gamma$ games are determinate.

$\Gamma$ determinacy in $X^{\mathbb{N}}: \operatorname{Det}^{X}[\varphi]$ for any $\Gamma$ game $\varphi(f)$ in $X^{\mathbb{N}}$.

The following $\Delta_{n}^{i}$ determinacy asserts the determinacies of games both $\Sigma_{n}^{i}$ and $\Pi_{n}^{i}$, in a sense. 
$\Delta_{n}^{i}$ determinacy in $X^{\mathbb{N}}: \forall f \in X^{\mathbb{N}}(\varphi(f) \leftrightarrow \psi(f)) \rightarrow \operatorname{Det}^{X}[\varphi]$

where $\varphi(f)$ is $\Sigma_{n}^{i}$ game and where $\psi(f)$ is a $\Pi_{n}^{i}$ games in $X^{\mathbb{N}}$.

Det* and Det abbreviate determinacy in the Cantor space and that in the Baire space, respectively.

In this paper, for every game $\varphi(f)$ in $X^{\mathbb{N}}$, we say player I (resp. II) wins $\varphi(f)$ at $s \in X^{<\mathbb{N}}$ if

(1) $|s|$ is even and player I (resp. II) has a winning strategy in $\varphi(s * f)$, or

(2) $|s|$ is odd and player II (resp. I) has a winning strategy in $\varphi(s * f)$.

Note that in case (2) players are exchanged.

\subsection{Wadge classes in descriptive set theory}

Here we review Wadge classes of Polish spaces, which gives us intuitive ideas for determinacy schemata defined in the next subsection. Note that, in this subsection, we work in the usual framework of (descriptive) set theory, not in that of second order arithmetic.

For a pair $(A, B)$ of subsets of a Polish space $X$, how can we define which set is "simpler"? Wadge reducibility gives an answer: We say that $A$ is Wadge reducible to $B$, in symbols $A \leq_{W} B$, if there is a continuous map $f: X \rightarrow X$ such that $f^{-1}(B)=A$. We write $A \equiv_{W} B$ if and only if $A \leq_{W} B$ and $B \leq_{W} A$. Clearly $\equiv_{W}$ is an equivalence relation, and associated equivalence classes $\mathbf{A}=[A]_{W}$ are called Wadge degrees. For a set $A \subseteq X^{\mathbb{N}}$, the coarse degree $\mathbf{A}^{*}$ of $A$ is $[A]_{W} \cup\left[A^{c}\right]_{W}$, where $A^{c}$ is the complement of $A$. WADGE denotes the set of the coarse degrees of Borel sets, and $\leq_{W}^{*}$ is defined by $\mathbf{A} \leq_{W}^{*} \mathbf{B} \leftrightarrow A \leq_{W} B \vee A \leq_{W} B^{c}$.

Under the assumption of Borel determinacy, which is proved in ZFC, (WADGE, $\leq_{W}^{*}$ ) is wellordered. Actually it gives a finer hierarchy than Hausdorff and Kuratowski's difference hierarchy from $[1, \S 37$. III. Theorem], the finite level of which is defined as follows:

- $\left(\boldsymbol{\Sigma}_{n}^{0}\right)_{1}=\boldsymbol{\Sigma}_{n}^{0}$

- $\left(\boldsymbol{\Sigma}_{n}^{0}\right)_{m+1}=\left\{A \backslash B: A \in \Sigma_{n}^{0}\right.$ and $\left.B \in\left(\boldsymbol{\Sigma}_{n}^{0}\right)_{m}\right\}$

A concrete description of the Wadge hierarchy of Borel sets is provided in [2]. The classes in Wadge hierarchy are composed from the classes of Hausdorff and Kuratowski's difference hierarchy by means of taking (countable) unions and complements. Especially, below $\left(\boldsymbol{\Sigma}_{2}^{0}\right)_{2}$, the construction principle is easier because we do not need countable unions.

Figure 2 is a rough sketch of Wadge hierarchy below $\left(\boldsymbol{\Sigma}_{2}^{0}\right)_{2}$, where only one of mutually dual classes is described, where $\operatorname{Sep}\left(\boldsymbol{\Delta}_{2}^{0}, \boldsymbol{\Sigma}_{2}^{0}\right)$ is the union of all Wadge classes below $\left(\boldsymbol{\Sigma}_{2}^{0}\right)_{2}$ and where $\boldsymbol{\Delta}_{2}^{0}$ is the union of all the Wadge classes below $\boldsymbol{\Sigma}_{2}^{0}$. The constructions mentioned here are as follows:

- $\boldsymbol{\Delta}(\boldsymbol{\Gamma})=\left\{A: A, A^{c} \in \boldsymbol{\Gamma}\right\}$

- $\operatorname{Sep}\left(\boldsymbol{\Gamma}, \boldsymbol{\Gamma}^{\prime}\right)=\left\{\left(A \cap B^{c}\right) \cup\left(A^{c} \cap C\right): A \in \boldsymbol{\Gamma}\right.$ and $\left.B, C \in \boldsymbol{\Gamma}^{\prime}\right\}$

- $\operatorname{Bisep}\left(\boldsymbol{\Gamma}, \boldsymbol{\Gamma}^{\prime}\right)=\left\{\left(A \cap B^{c}\right) \cup(C \cap D): A, C \in \boldsymbol{\Gamma}, A \cap C=\emptyset\right.$ and $\left.B, D \in \boldsymbol{\Gamma}^{\prime}\right\}$

Then we can easily construct corresponding schemata of determinacy; replacing $\cup$ with $\vee$ and replacing $(-)^{c}$ (complement) with $\neg$. The definitions of $\operatorname{Sep}\left(\Gamma, \Gamma^{\prime}\right)$ and $\operatorname{Bisep}\left(\Gamma, \Gamma^{\prime}\right)$ determinacies in the next subsection are motivated by these constructions of Wadge classes in this way.

In this paper, we treat $\operatorname{Bisep}\left(\Delta_{1}^{0}, \Sigma_{1}^{0}\right), \operatorname{Bisep}\left(\Delta_{1}^{0}, \Sigma_{2}^{0}\right), \operatorname{Bisep}\left(\Sigma_{1}^{0}, \Sigma_{2}^{0}\right), \operatorname{Sep}\left(\Sigma_{1}^{0}, \Sigma_{2}^{0}\right)$ and $\operatorname{Bisep}\left(\Delta_{2}^{0}, \Sigma_{2}^{0}\right)$ determinacies, which correspond to $\operatorname{Bisep}\left(\boldsymbol{\Delta}_{1}^{0}, \boldsymbol{\Sigma}_{1}^{0}\right), \operatorname{Bisep}\left(\boldsymbol{\Delta}_{1}^{0}, \boldsymbol{\Sigma}_{2}^{0}\right), \operatorname{Bisep}\left(\boldsymbol{\Sigma}_{1}^{0}, \boldsymbol{\Sigma}_{2}^{0}\right), \operatorname{Sep}\left(\boldsymbol{\Sigma}_{1}^{0}, \boldsymbol{\Sigma}_{2}^{0}\right)$ and $\operatorname{Bisep}\left(\boldsymbol{\Delta}_{2}^{0}, \boldsymbol{\Sigma}_{2}^{0}\right)$ respectively. In section 5 , we show that $\Pi_{1}^{1}$ comprehension, $\operatorname{Bisep}\left(\Sigma_{1}^{0}, \Sigma_{2}^{0}\right)$-Det ${ }^{*}$ and $\operatorname{Sep}\left(\Sigma_{1}^{0}, \Sigma_{2}^{0}\right)$-Det* are pairwise equivalent. Although there are $\omega_{1}$ different Wadge classes between $\operatorname{Bisep}\left(\Sigma_{1}^{0}, \boldsymbol{\Sigma}_{1}^{0}\right)$ and $\operatorname{Sep}\left(\boldsymbol{\Sigma}_{1}^{0}, \boldsymbol{\Sigma}_{2}^{0}\right)$, we will show that, in the Cantor space, the strengths of the determinacies correspond to them coincide. 


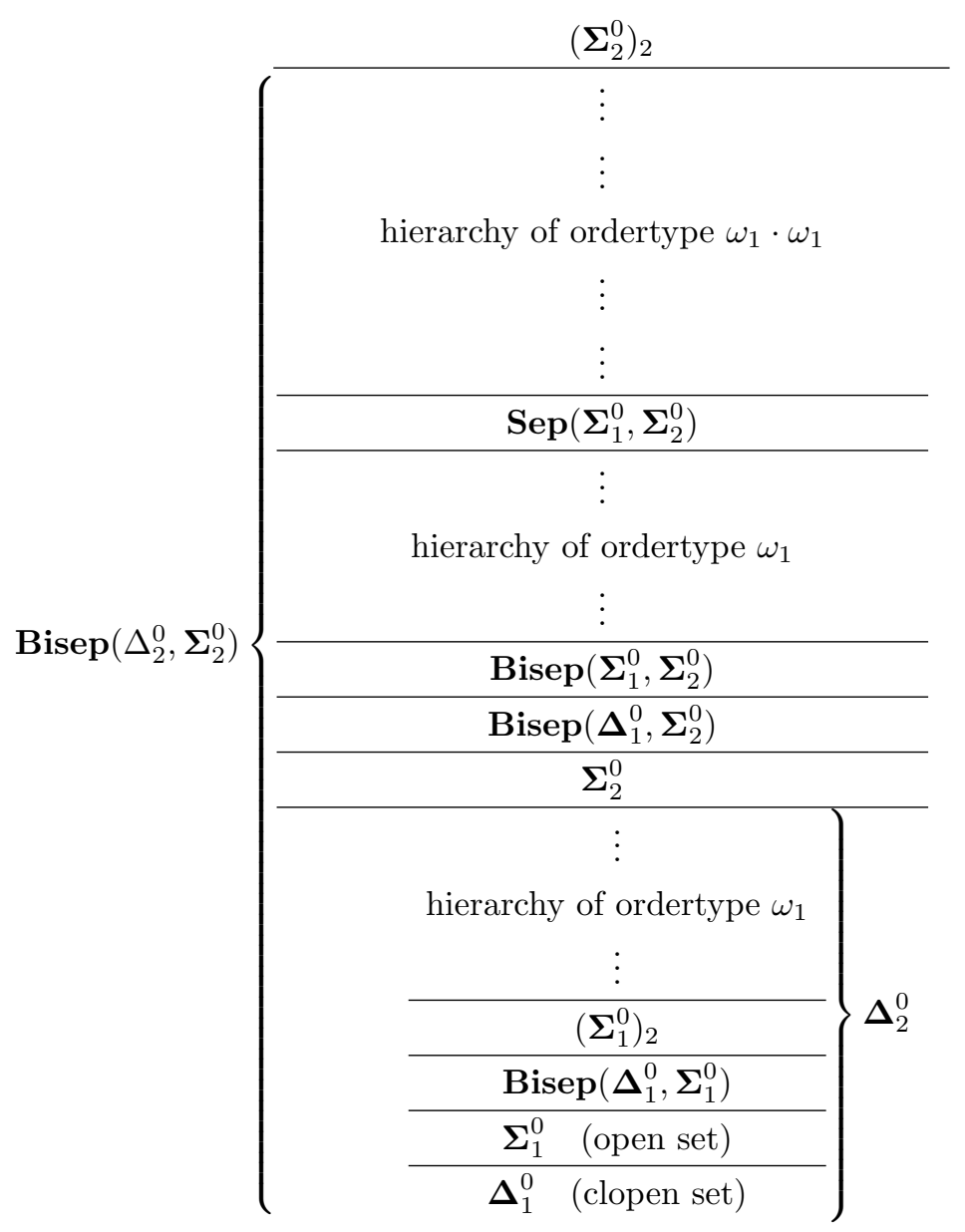

Figure 2: Wadge hierarchy below $\left(\Sigma_{2}^{0}\right)_{2}$ due to [2]

\subsection{Several new determinacy schemata}

In this subsection we define, in second order arithmetic, determinacy schemata which correspond to the determinacy of Wadge classes in descriptive set theory. We only consider classes of formulae $\varphi(f)$ with a distinguished function variable $f \in X^{\mathbb{N}}$.

The normal form theorem for $\Sigma_{1}^{0}$ formulae over $\mathrm{RCA}_{0}$ is proved in [8]. Here we prove it over $\mathrm{RCA}_{0}^{*}$.

While we usually assume that formulae and terms may contain free variables other than explicitly displayed, only in the statement and the proof of the following lemma, we assume that all free variables of formulae and terms are among displayed ones.

Lemma 2.13 (normal form theorem 1) For any $\Sigma_{1}^{0}$ formula $\varphi(f, \vec{k}, \vec{Y})$ with a distinguished function variable $f \in 2^{\mathbb{N}}$, we can find a $\Pi_{0}^{0}$ formula $\theta(s, \vec{k}, \vec{Y})$, in which $f$ does not occur freely, such that $\mathrm{RCA}_{0}^{*}$ proves $\forall f \in 2^{\mathbb{N}} \forall \vec{k} \forall \vec{Y}(\varphi(f, \vec{k}, \vec{k}, \vec{Y}) \leftrightarrow \exists n \theta(f[n], \vec{k}, \vec{Y}))$.

Proof. First, we prove that, for every $\Pi_{0}^{0}$ formula $\varphi(f, \vec{k}, \vec{Y})$, we can find $\Pi_{0}^{0}$ formula $\theta(s, \vec{k}, \vec{Y})$ and a term $t(\vec{k})$ such that $\operatorname{RCA}_{0}^{*}$ proves $\varphi(f, \vec{k}, \vec{Y}) \leftrightarrow \theta(f[t(\vec{k})], \vec{k}, \vec{Y})$ by induction on construction of formula. The cases of atomic formulae, Boolean operations. The case of bounded universal quantifier can be proved as follows: Let $\varphi(f, \vec{k}, \vec{Y})$ be $\Pi_{0}^{0}$ of the form $\forall i<t^{\prime}(\vec{k}) \theta^{\prime}(i, f, \vec{k}, \vec{Y})$, where $\theta^{\prime}(x, f, \vec{k}, \vec{Y})$ is $\Pi_{0}^{0}$. By induction hypothesis, we can find a $\Pi_{0}^{0}$ formula $\theta^{\prime \prime}(x, s, \vec{k}, \vec{Y})$ and a term $t^{\prime \prime}(i, \vec{k})$ such that 
$\mathrm{RCA}_{0}^{*}$ proves $\theta^{\prime}(i, f, \vec{k}, \vec{Y}) \leftrightarrow \theta^{\prime \prime}\left(i, f\left[t^{\prime \prime}(i, \vec{k})\right], \vec{k}, \vec{Y}\right)$. Then, we can check that $\mathrm{RCA}_{0}^{*}$ proves

$$
\begin{aligned}
& \forall i<t^{\prime}(\vec{k}) \theta^{\prime}(i, f, \vec{k}, \vec{Y}) \\
\leftrightarrow \quad & \forall i<t^{\prime}(\vec{k}) \theta^{\prime \prime}\left(i, f\left[t^{\prime \prime}(i, \vec{k})\right], \vec{k}, \vec{Y}\right) \\
\leftrightarrow \quad & \forall i<t^{\prime}(\vec{k}) \theta^{\prime \prime}\left(i,\left(f\left[t^{\prime \prime}\left(t^{\prime}(\vec{k}), \vec{k}\right)\right]\right)[t(i, \vec{k})], \vec{k}, \vec{Y}\right),
\end{aligned}
$$

by the monotony of the term. The case of bounded existential quantifier can be proved similarly.

Now we complete the proof. Let $\varphi(f, \vec{k}, \vec{Y}) \equiv \exists n \theta^{\prime}(n, f, \vec{k}, \vec{Y})$ for some $\Pi_{0}^{0}$ formula $\theta^{\prime}(x, f, \vec{k}, \vec{Y})$. By the above, we can fine $\Pi_{0}^{0}$ formula $\theta^{\prime \prime}(x, f, \vec{k}, \vec{Y})$ and a term $t^{\prime}(\vec{k})$ such that $\operatorname{RCA}_{0}^{*}$ proves $\theta^{\prime}(x, f, \vec{k}, \vec{Y}) \leftrightarrow$ $\theta^{\prime \prime}(x, f[t(x, \vec{k})], \vec{k}, \vec{Y})$. Then $\mathrm{RCA}_{0}^{*}$ proves

$$
\begin{aligned}
& \varphi(f, \vec{k}, \vec{Y}) \\
\leftrightarrow & \exists n \theta^{\prime \prime}(n, f[t(n, \vec{k})], \vec{k}, \vec{Y}) \\
\leftrightarrow & \exists n \exists n^{\prime}<n+1\left(n=\left\langle n^{\prime}, t\left(n^{\prime}, \vec{k}\right)\right\rangle \wedge \theta^{\prime \prime}\left(n^{\prime},(f[n])\left[t\left(n^{\prime}, \vec{k}\right)\right], \vec{k}, \vec{Y}\right)\right) .
\end{aligned}
$$

Definition 2.14 Let $\Gamma$ and $\Gamma^{\prime}$ be classes of formulae. $\Gamma \wedge \Gamma^{\prime}$ (resp. $\Gamma \vee \Gamma^{\prime}$ ) is the class of formulae of the form $\varphi_{0} \wedge \varphi_{1}$ (resp. $\varphi_{0} \vee \varphi_{1}$ ), where $\varphi_{0}$ is $\Gamma$ and $\varphi_{1}$ is $\Gamma^{\prime} . \neg \Gamma$ is the class of formulae of the form $\neg \varphi$, where $\varphi$ is $\Gamma$.

We consider determinacy schemata also for the class defined in Definition 2.14. Furthermore, we treat the following determinacy schemata, which are motivated by Borel Wadge classes, mentioned in the previous subsection.

\section{Definition 2.15}

$\Delta(\Gamma)$ determinacy in $X^{\mathbb{N}}: \forall f \in X^{\mathbb{N}}(\psi(f) \leftrightarrow \xi(f)) \rightarrow \operatorname{Det}^{X}[\psi]$, where $\psi(f)$ is $\Gamma$ and where $\xi(f)$ is $\neg \Gamma$.

$\operatorname{Sep}\left(\Sigma_{n}^{0}, \Sigma_{m}^{0}\right)$ determinacy in $X^{\mathbb{N}}: \operatorname{Det}^{X}\left[\left(\psi \wedge \eta_{0}\right) \vee\left(\neg \psi \wedge \eta_{1}\right)\right]$, where $\psi(f)$ is $\Sigma_{n}^{0}$, where $\eta_{0}(f)$ is $\Pi_{m}^{0}$ and where $\eta_{1}(f)$ is $\Sigma_{m}^{0}$.

$\operatorname{Sep}\left(\Delta_{n}^{0}, \Sigma_{m}^{0}\right)$ determinacy in $X^{\mathbb{N}}: \forall f \in X^{\mathbb{N}}(\psi(f) \leftrightarrow \xi(f)) \rightarrow \operatorname{Det}^{X}\left[\left(\psi \wedge \eta_{0}\right) \vee\left(\neg \psi \wedge \eta_{1}\right)\right]$, where $\psi(f)$ is $\Sigma_{n}^{0}$, where $\xi(f)$ is $\Pi_{n}^{0}$, where $\eta_{0}(f)$ is $\Pi_{m}^{0}$ and where $\eta_{1}(f)$ is $\Sigma_{m}^{0}$.

$\operatorname{Bisep}\left(\Sigma_{n}^{0}, \Sigma_{m}^{0}\right)$ determinacy in $X^{\mathbb{N}}: \forall f \in X^{\mathbb{N}} \neg\left(\psi_{0}(f) \wedge \psi_{1}(f)\right) \rightarrow \operatorname{Det}^{X}\left[\left(\psi_{0} \wedge \eta_{0}\right) \vee\left(\psi_{1} \wedge \eta_{1}\right)\right]$, where $\psi_{i}(f)$ 's are $\Sigma_{n}^{0}$, where $\eta_{0}(f)$ is $\Pi_{m}^{0}$ and where $\eta_{1}(f)$ is $\Sigma_{m}^{0}$.

$\operatorname{Bisep}\left(\Delta_{n}^{0}, \Sigma_{m}^{0}\right)$ determinacy in $X^{\mathbb{N}}$ :

$$
\begin{aligned}
\forall f \in X^{\mathbb{N}}\left(\neg\left(\psi_{0}(f) \wedge \psi_{1}(f)\right)\right) \wedge \forall f \in X^{\mathbb{N}}\left(\psi_{0}(f) \leftrightarrow \xi_{0}(f)\right) \wedge \forall f & \in X^{\mathbb{N}}\left(\psi_{1}(f) \leftrightarrow \xi_{1}(f)\right) \\
& \rightarrow \operatorname{Det}^{X}\left[\left(\psi_{0} \wedge \eta_{0}\right) \vee\left(\psi_{1} \wedge \eta_{1}\right)\right],
\end{aligned}
$$

where $\psi_{i}(f)$ 's are $\Sigma_{n}^{0}$, where $\xi_{i}(f)$ 's are $\Pi_{n}^{0}$, where $\eta_{0}(f)$ is $\Pi_{m}^{0}$ and where $\eta_{1}(f)$ is $\Sigma_{m}^{0}$.

Remark 2.16 $\operatorname{Bisep}\left(\Delta_{n}^{0}, \boldsymbol{\Sigma}_{n+m}^{0}\right)=\operatorname{Sep}\left(\Delta_{n}^{0}, \boldsymbol{\Sigma}_{n+m}^{0}\right)$ in the terms of the last subsection. Inspired by this equality, we can see that $\operatorname{Bisep}\left(\Delta_{n}^{0}, \Sigma_{n+m}^{0}\right)$ determinacy in $X^{\mathbb{N}}$ is equivalent to $\operatorname{Sep}\left(\Delta_{n}^{0}, \Sigma_{n+m}^{0}\right)$ determinacy in $X^{\mathbb{N}}$ over $\mathrm{RCA}_{0}^{*}$, since, for any formulae $\psi_{0}(f), \psi_{1}(f), \eta_{0}(f)$ and $\eta_{1}(f), \mathrm{RCA}_{0}^{*}$ proves the following:

$$
\begin{aligned}
& \forall f \in X^{\mathbb{N}} \neg\left(\psi_{0}(f) \wedge \psi_{1}(f)\right) \rightarrow \\
& \quad \forall f \in X^{\mathbb{N}}\left(\left(\left(\psi_{0}(f) \wedge \eta_{0}(f)\right) \vee\left(\psi_{1}(f) \wedge \eta_{1}(f)\right)\right) \leftrightarrow\left(\left(\psi_{0}(f) \wedge \eta_{0}(f)\right) \vee\left(\neg \psi_{0}(f) \wedge\left(\psi_{1}(f) \wedge \eta_{1}(f)\right)\right)\right)\right) .
\end{aligned}
$$


The next lemma is a reformulation of a classical separation theorem: For any disjoint pair of $\mathbf{\Pi}_{n}^{0}$ sets $A$ and $B$, there exists a $\boldsymbol{\Delta}_{n}^{0}$ set $C$ such that $A \subseteq C$ and $B \subseteq C^{c}$.

Lemma 2.17 Assume $0<n<\omega$. For any pair of $\Pi_{n}^{0}$ formulae $\varphi_{0}(f)$ and $\varphi_{1}(f)$, we can find a $\Sigma_{n}^{0}$ formula $\psi(f)$ and $\Pi_{n}^{0}$ formula $\xi(f)$ such that $\mathrm{RCA}_{0}^{*}$ proves

$$
\forall f \in X^{\mathbb{N}} \neg\left(\varphi_{0}(f) \wedge \varphi_{1}(f)\right) \rightarrow \forall f \in X^{\mathbb{N}}\left((\psi(f) \leftrightarrow \xi(f)) \wedge\left(\varphi_{0}(f) \rightarrow \psi(f)\right) \wedge\left(\varphi_{1}(f) \rightarrow \neg \psi(f)\right)\right)
$$

Proof. Let $\varphi_{i}(f)$ be $\Pi_{n}^{0}$, say $\forall m \theta_{i}(m, f)$, where $\theta_{i}(x, g)$ is $\Sigma_{n-1}^{0}$. Assume $\forall f \in X^{\mathbb{N}} \neg\left(\varphi_{0}(f) \wedge \varphi_{1}(f)\right)$. Set $\psi(f) \equiv \exists m\left(\neg \theta_{1}(m, f) \wedge \forall l<m \theta_{0}(l, f)\right)$. Then we easily see that $\varphi_{0}(f) \rightarrow \psi(f)$ and $\varphi_{1}(f) \rightarrow$ $\neg \psi(f)$ and that $\psi(f) \leftrightarrow \forall m\left(\theta_{0}(m, f) \vee \exists l \leq m \neg \theta_{1}(l, f)\right)$.

The following lemma is useful to investigate the relationship among determinacy schemata defined in 2.15. The motivating result is $\operatorname{Sep}\left(\boldsymbol{\Delta}_{n}^{0}, \boldsymbol{\Sigma}_{n}^{0}\right)=\boldsymbol{\Delta}\left(\left(\boldsymbol{\Sigma}_{n}^{0}\right)_{2}\right)=\boldsymbol{\Delta}\left(\boldsymbol{\Sigma}_{n}^{0} \wedge \boldsymbol{\Pi}_{n}^{0}\right)$.

Lemma 2.18 Let $0<n<\omega$.

1. For any $\Sigma_{n}^{0}$ formulae $\psi_{0}(f)$ and $\eta_{1}(f)$, for any $\Pi_{n}^{0}$ formulae $\psi_{1}(f)$ and $\eta_{0}(f)$, we can find $\Sigma_{n}^{0}$ formulae $\xi_{0}(f)$ and $\xi_{1}(f)$ and $\Pi_{n}^{0}$ formulae $\zeta_{0}(f)$ and $\zeta_{1}(f)$ such that $\mathrm{RCA}_{0}^{*}$ proves

$$
\begin{aligned}
& \forall f \in X^{\mathbb{N}}\left(\psi_{0}(f) \leftrightarrow \psi_{1}(f)\right) \rightarrow \\
& \quad \forall f \in X^{\mathbb{N}}\left(\left(\left(\psi_{0}(f) \wedge \eta_{0}(f)\right) \vee\left(\psi_{1}(f) \wedge \eta_{1}(f)\right)\right) \leftrightarrow\left(\xi_{0}(f) \wedge \zeta_{0}(f)\right) \leftrightarrow\left(\xi_{1}(f) \vee \zeta_{1}(f)\right)\right)
\end{aligned}
$$

2. For any $\Sigma_{n}^{0}$ formulae $\xi_{0}(f)$ and $\xi_{1}(f)$ and for any $\Pi_{n}^{0}$ formulae $\zeta_{0}(f)$ and $\zeta_{1}(f)$, we can find $\Sigma_{n}^{0}$ formulae $\psi_{0}(f)$ and $\eta_{1}(f)$ and $\Pi_{n}^{0}$ formulae $\psi_{1}(f)$ and $\eta_{0}(f)$ such that $\mathrm{RCA}_{0}^{*}$ proves

$$
\begin{aligned}
& \forall f \in X^{\mathbb{N}}\left(\left(\xi_{0}(f) \wedge \zeta_{0}(f)\right) \leftrightarrow\left(\xi_{1}(f) \vee \zeta_{1}(f)\right)\right) \rightarrow \\
& \forall f \in X^{\mathbb{N}}\left(\psi_{0}(f) \leftrightarrow \psi_{1}(f)\right) \wedge \forall f \in X^{\mathbb{N}}\left(\left(\xi_{0}(f) \wedge \zeta_{0}(f)\right) \leftrightarrow\left(\left(\psi_{0}(f) \wedge \eta_{0}(f)\right) \vee\left(\neg \psi_{0}(f) \wedge \eta_{1}(f)\right)\right)\right)
\end{aligned}
$$

Proof. We work in $\mathrm{RCA}_{0}^{*}$.

(1) Assume that $\forall f \in X^{\mathbb{N}}\left(\psi_{0}(f) \leftrightarrow \psi_{1}(f)\right)$ for a $\Sigma_{n}^{0}$ formula $\psi_{0}(f)$ and a $\Pi_{n}^{0}$ formula $\psi_{1}(f)$. Then, it is easy to find $\Sigma_{n}^{0}$ formulae $\xi_{0}(f)$ and $\xi_{1}(f)$ and $\Pi_{n}^{0}$ formulae $\zeta_{0}(f)$ and $\zeta_{1}(f)$ such that $\mathrm{RCA}_{0}^{*}$ proves

$$
\begin{gathered}
\forall f \in X^{\mathbb{N}}\left(\xi_{0}(f) \leftrightarrow\left(\psi_{0}(f) \vee\left(\neg \psi_{0}(f) \wedge \eta_{1}(f)\right)\right), \quad \forall f \in X^{\mathbb{N}}\left(\xi_{1}(f) \leftrightarrow\left(\neg \psi_{0}(f) \wedge \eta_{1}(f)\right)\right),\right. \\
\forall f \in X^{\mathbb{N}}\left(\zeta_{0}(f) \leftrightarrow\left(\left(\psi_{0}(f) \wedge \eta_{0}(f)\right) \vee \neg \psi_{0}(f)\right)\right), \quad \forall f \in X^{\mathbb{N}}\left(\zeta_{1}(f) \leftrightarrow\left(\psi_{0}(f) \wedge \eta_{0}(f)\right)\right),
\end{gathered}
$$

and they enjoy the desired property.

\begin{tabular}{|c|c|c|}
\hline$\neg \xi_{0}(f)$ & $\neg \xi_{0}(f) \wedge \neg \zeta_{0}(f)$ & $\neg \zeta_{0}(f)$ \\
\hline$\xi_{1}(f)$ & $\xi_{1}(f) \wedge \zeta_{1}(f)$ & $\zeta_{1}(f)$ \\
\hline
\end{tabular}

(2) Assume $\forall f \in X^{\mathbb{N}}\left(\left(\xi_{0}(f) \wedge \zeta_{0}(f)\right) \leftrightarrow\left(\xi_{1}(f) \vee \zeta_{1}(f)\right)\right)$ for $\Sigma_{n}^{0}$ formulae $\xi_{0}(f)$ and $\xi_{1}(f)$ and $\Pi_{n}^{0}$ formulae $\zeta_{0}(f)$ and $\zeta_{1}(f)$. Note that $\forall f \in X^{\mathbb{N}}\left(\left(\neg \xi_{0}(f) \vee \neg \zeta_{0}(f)\right) \leftrightarrow \neg\left(\xi_{1}(f) \vee \zeta_{1}(f)\right)\right)$, and so there is no $f \in X^{\mathbb{N}}$ with $\neg \xi_{0}(f) \wedge \zeta_{1}(f)$. By Lemma 2.17, we can find a $\Sigma_{n}^{0}$ formula $\psi_{0}(f)$ and a $\Pi_{n}^{0}$ formula $\psi_{1}(f)$ such that

$$
\forall f \in X^{\mathbb{N}}\left(\left(\psi_{0}(f) \leftrightarrow \psi_{1}(f)\right) \wedge\left(\zeta_{1}(f) \rightarrow \psi_{0}(f)\right) \wedge\left(\neg \xi_{0}(f) \rightarrow \neg \psi_{0}(f)\right)\right) .
$$

$$
\begin{array}{|l|l|l|}
\hline \multicolumn{3}{|c|}{\psi_{1}(f)} \\
\hline \xi_{0}(f) & \neg \xi_{0}(f) \wedge \neg \zeta_{0}(f) & \neg \zeta_{0}(f) \\
\hline \xi_{1}(f) & \xi_{1}(f) \wedge \zeta_{1}(f) & \zeta_{1}(f) \\
\hline
\end{array}
$$

Then we can check

$$
\forall f \in X^{\mathbb{N}}\left(\left(\xi_{0}(f) \wedge \zeta_{0}(f)\right) \leftrightarrow\left(\left(\psi_{0}(f) \wedge \zeta_{0}(f)\right) \vee\left(\neg \psi_{0}(f) \wedge \xi_{1}(f)\right)\right)\right)
$$


and so $\psi_{i}(f)$ 's, $\eta_{0}(f) \equiv \zeta_{0}(f)$ and $\eta_{1}(f) \equiv \xi_{1}(f)$ enjoy the desired property.

By Remark 2.16 and Lemma 2.18, $\operatorname{Bisep}\left(\Delta_{n}^{0}, \Sigma_{n}^{0}\right)$-Det, $\operatorname{Sep}\left(\Delta_{n}^{0}, \Sigma_{n}^{0}\right)$-Det and $\Delta\left(\Sigma_{n}^{0} \wedge \Pi_{n}^{0}\right)$-Det are pairwise equivalent over $\operatorname{RCA}_{0}^{*}$ and $\operatorname{Bisep}\left(\Delta_{n}^{0}, \Sigma_{n}^{0}\right)$-Det ${ }^{*}, \operatorname{Sep}\left(\Delta_{n}^{0}, \Sigma_{n}^{0}\right)$-Det* and $\Delta\left(\Sigma_{n}^{0} \wedge \Pi_{n}^{0}\right)$-Det* are pairwise equivalent over $\mathrm{RCA}_{0}^{*}$.

\section{Weak König's lemma and determinacy}

In this section, we investigate the relation among determinacy, weak König's lemma and $\Sigma_{1}^{0}$ induction.

[5] shows that $\Delta_{1}^{0}$-Det* $\Sigma_{1}^{0}$-Det* and weak König's lemma are pairwise equivalent over RCA $A_{0}$. However, it seems that their proofs do not essentially require $\Sigma_{1}^{0}$ induction. In order to make it precise, we need to consider the system $W K L_{0}$ minus $\Sigma_{1}^{0}$ induction, i.e., $W K L_{0}^{*}$. Then we have the following:

Proposition 3.1 RCA $\vdash \Delta_{1}^{0}$-Det ${ }^{*} \leftrightarrow \Sigma_{1}^{0}$-Det $^{*} \leftrightarrow \mathrm{WKL}_{0}^{*}$.

Proof. See [5]. Although the original proofs show the equivalence over $\mathrm{RCA}_{0}$, it can be replaced with $\mathrm{RCA}_{0}^{*}$. Note that $\Sigma_{1}^{0}$ separation is equivalent to weak König's lemma over RCA $A_{0}^{*}$.

Next we consider the strength of $\Sigma_{1}^{0}$ induction+weak König's lemma over RCA $A_{0}^{*}$ For that purpose, we introduce a set comprehension axiom which is equivalent to $\Sigma_{1}^{0}$ induction.

Lemma 3.2 ([8, Theorem II.3.8, Theorem X.4.4]) $\Sigma_{1}^{0}$ induction is equivalent to bounded $\Sigma_{1}^{0}$ comprehension over $\mathrm{RCA}_{0}^{*}$.

The theorems below show $\Sigma_{1}^{0}$ induction+weak König's lemma is equivalent to $\operatorname{Bisep}\left(\Delta_{1}^{0}, \Sigma_{1}^{0}\right)$-Det* over $\mathrm{RCA}_{0}^{*}$.

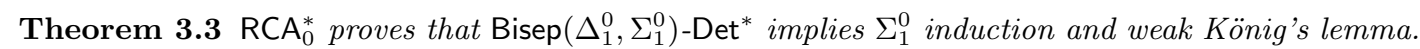

Proof. Assume $\operatorname{Bisep}\left(\Delta_{1}^{0}, \Sigma_{1}^{0}\right)$-Det* ${ }^{*}$ Since $\operatorname{Bisep}\left(\Delta_{1}^{0}, \Sigma_{1}^{0}\right)$-Det* implies $\Sigma_{1}^{0}$-Det* and so weak König's lemma, it is enough to prove bounded $\Sigma_{1}^{0}$ comprehension, which is equivalent to $\Sigma_{1}^{0}$ induction by Lemma 3.2. Let $\varphi(x)$ be a $\Sigma_{1}^{0}$ formula. For fixed $n$, we consider the following game. Player I chooses $k<n$ and asks whether $\varphi(k)$ or not. Player II answers yes or no. She wins if and only if her answer is correct. Such a game is realized as follows:

- Player I chooses $k<n$ by playing 0 at his first $k$ turns and 1 at his $(k+1)$-th turn.

- When player II chooses 1 at her $(k+1)$-th turn, she wins if $\varphi(k)$ holds.

- When player II chooses 0 at her $(k+1)$-th turn, she wins if $\neg \varphi(k)$ holds.

The game goes as follows:

$$
\begin{array}{cccc}
\text { player I } & \overbrace{0, \ldots, 0,}^{k \text { times }} & 1 & \ldots \\
\text { player II } & *, \ldots, * & \underbrace{i}_{\text {answer }} & \ldots
\end{array}
$$

Player I wins if and only if one of the following holds:

- Player I chooses $k<n$, player II answers "yes," and $\neg \varphi(k)$ holds.

- Player I chooses $k<n$, player II answers "no," and $\varphi(k)$ holds.

Formally, this winning condition can be written as below:

$$
(\exists k<n(\psi(f, k, 1) \wedge \neg \varphi(k))) \vee(\exists k<n(\psi(f, k, 0) \wedge \varphi(k))),
$$


where $\psi(f, k, i)$ is the $\Pi_{0}^{0}$ formula $\forall i<k(f(2 i)=0) \wedge f(2 k)=1 \wedge f(2 k+1)=i$. (b) is equivalent to the following formula:

$(\underbrace{(\exists k<n \psi(f, k, 1))}_{(1)} \wedge \underbrace{\forall k<n(\psi(f, k, 1) \rightarrow \neg \varphi(k))}_{(2)}) \vee(\underbrace{(\exists k<n \psi(f, k, 0))}_{(3)} \wedge \underbrace{\exists k<n(\psi(f, k, 0) \wedge \varphi(k))}_{(4)})$.

Clearly (1) and (3) are $\Sigma_{1}^{0}$, and we can easily see that (1) and (3) are equivalent to some $\Pi_{1}^{0}$ formulae. There is no $f \in 2^{\mathbb{N}}$ which satisfies both (1) and (3). (2) is equivalent to some $\Pi_{1}^{0}$ formula and (4) is equivalent to some $\Sigma_{1}^{0}$ formula. Player I has no winning strategy in (b), since for any $k<m$, player I cannot win if player II's answer is correct. By $\operatorname{Bisep}\left(\Delta_{1}^{0}, \Sigma_{1}^{0}\right)$-Det*, player II has a winning strategy $\tau$ in (b). We can also see that, $\tau\left(k^{\tau} *\langle 1\rangle\right)=1$ if and only if $\varphi(k)$ holds for any $k<n$,

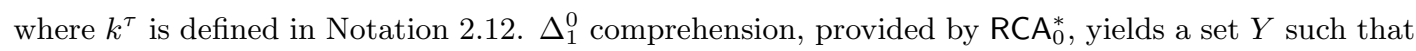
$k \in Y \leftrightarrow k<n \wedge \tau\left(k^{\tau} *\langle 1\rangle\right)=1$. Intuitively, $Y$ is the set of natural numbers $k<n$ such that the winning strategy $\tau$ tells player II to answer "yes" when she is asked about it. We can check that $\forall k(\varphi(k) \wedge k<n \leftrightarrow k \in Y)$

For the converse, we must notice that the class of $\Pi_{1}^{0}$ formulae is enriched by weak König's lemma.

Lemma 3.4 For a $\Pi_{1}^{0}$ formula $\varphi(X)$, there is a $\Pi_{1}^{0}$ formula $\widehat{\varphi}$ such that $\mathrm{WKL}_{0}^{*}$ proves $\widehat{\varphi} \leftrightarrow \exists X \varphi(X)$.

Proof. See [8, Lemma VIII.2.4]. Although the original lemma is proved in $\mathrm{WKL}_{0}$, we can check that the proof works also in $\mathrm{WKL}_{0}^{*}$.

Remark 3.5 1. For a $\Pi_{1}^{0}$ game $\varphi(f)$ in the Cantor space, the assertion " $\sigma$ is a winning strategy for player I in $\varphi(f)$ " is equivalent to a $\Pi_{1}^{0}$ formula over $\operatorname{RCA}_{0}^{*}$. Let $\varphi(f) \equiv \forall n \theta(f[n])$. Then this assertion can be written as $\forall \tau \in \mathcal{S}_{\mathrm{II}}^{\{0,1\}} \forall n \theta(\sigma \otimes \tau[n])$, which is equivalent to a $\Pi_{1}^{0}$ formula $\forall s \in 2^{<\mathbb{N}} \forall n<2|s| \theta((\sigma \otimes s)[n])$, where $\sigma \otimes s$ is the sequence of length $2|s|$ such that, for any $2 k<2|s|,(\sigma \otimes s)(2 k)=\sigma((\sigma \otimes s)[2 k])$ and, for each $2 k+1<2|s|,(\sigma \otimes s)(2 k+1)=s(k)$.

2. For a $\Sigma_{1}^{0}$ game $\varphi(f)$ in the Cantor space, the assertion " $\sigma$ is a winning strategy for player I in $\varphi(f)$ " is equivalent to a $\Sigma_{1}^{0}$ formula over $\mathrm{WKL}_{0}^{*}$. Say $\varphi(f) \equiv \exists n \theta(f[n])$. For a strategy $\sigma$ for player I, $T_{\sigma}=\left\{s \in 2^{<\mathbb{N}}: \forall 2 n<|s|(\sigma(s[2 n])=s(2 n) \wedge \forall t \subseteq s \neg \theta(t))\right\}$ is a tree. By weak König's lemma, the assertion is equivalent to the finiteness of $T_{\sigma}$, which can be written in $\Sigma_{1}^{0}$.

3. For any $\Pi_{1}^{0}$ game $\varphi(f)$ in the Cantor space, the assertion "player I has a winning strategy in $\varphi(f)$ " is equivalent to a $\Pi_{1}^{0}$ formula over $\mathrm{WKL}_{0}^{*}$. By 1 , the assertion can be written as $\exists \sigma \in \mathcal{S}_{\mathrm{I}}^{\{0,1\}} \psi(\sigma)$, where $\psi(X)$ is $\Pi_{1}^{0}$. This is equivalent to a $\Pi_{1}^{0}$ formula in $\mathrm{WKL}_{0}^{*}$ by Lemma 3.4 .

4. For any $\Sigma_{1}^{0}$ game $\varphi(f)$ in the Cantor space, the assertion "player I has a winning strategy in $\varphi(f)$ " is equivalent to a $\Sigma_{1}^{0}$ formula over $\mathrm{WKL}_{0}^{*}$. By a similar argument to 3 , the assertion "player II has a winning strategy in $\varphi(f)$ " is equivalent to a $\Pi_{1}^{0}$ formula $\psi$ over $\mathrm{WKL}_{0}^{*}$. By $\Sigma_{1}^{0}$ determinacy, which is proved in $\mathrm{WKL}_{0}^{*}$, the aimed assertion is equivalent to $\neg \psi$, and is $\Sigma_{1}^{0}$.

Lemma 3.6 (1) $\mathrm{RCA}_{0}^{*}$ proves $\Sigma_{1}^{0}$ axiom of choice. (2) $\mathrm{WKL}_{0}^{*}$ proves $\Pi_{1}^{0}$ axiom of choice.

Proof. (1) We work in $\mathrm{RCA}_{0}^{*}$. Assume $\forall n \exists Y \exists m \theta(n, Y[m])$, where $\theta(x, s)$ is $\Pi_{0}^{0}$. Then, by $\Delta_{1}^{0}$ comprehension, define $Z=\{\langle n, k\rangle: \theta(n, e(k))\}$, where $e: \mathbb{N} \rightarrow 2^{<\mathbb{N}}$ is the canonical enumeration of $2^{<\mathbb{N}}$. By the assumption $\forall n \exists Y \exists m \theta(n, Y[m])$, for each $n$, there is $k$ with $\langle n, k\rangle \in Z$. Take $f: \mathbb{N} \rightarrow \mathbb{N}$ such that $f(n)$ is the least $k$ such that $\langle n, k\rangle \in Z$ holds for all $n$. Clearly $Y=\{\langle n, m\rangle: m<$ $|e(f(n))| \wedge e(f(n))(m)=1\}$ enjoys the desired property.

(2) We work in $\mathrm{WKL}_{0}^{*}$. Assume $\forall n \exists Y \forall m \theta_{1}(n, Y[m])$, where $\theta_{1}(x, s)$ is $\Pi_{0}^{0}$. Define $T$ by $\left\{s \in 2^{<\mathbb{N}}:\right.$ $\left.\forall\langle n, m\rangle<|s| \theta_{1}\left(n, s_{n, m}\right)\right\}$, where $s_{n, m}=\langle s(\langle n, 0\rangle), s(\langle n, 1\rangle), \ldots, s(\langle n, m-1\rangle)\rangle$. Then $T$ is infinite if 
and only if $\forall n \exists Y \forall m \theta_{1}(n, Y[m])$. Using weak König's lemma, take a path $f$ of $T$. Then $Y=\{\langle n, m\rangle$ : $f(\langle n, m\rangle)=1\}$ enjoys the desired property.

Theorem 3.7 WKL $\mathrm{W}_{0}$ proves $\operatorname{Bisep}\left(\Delta_{1}^{0}, \Sigma_{1}^{0}\right)$-Det*

Proof. By Remark 2.16, it suffices to prove $\operatorname{Sep}\left(\Delta_{1}^{0}, \Sigma_{1}^{0}\right)$-Det* in WKL $\mathrm{L}_{0}$, instead of $\operatorname{Bisep}\left(\Delta_{1}^{0}, \Sigma_{1}^{0}\right)$ Det*. Let $\varphi(f)$ be a game of the form $\left(\psi(f) \wedge \eta_{0}(f)\right) \vee\left(\neg \psi(f) \wedge \eta_{1}(f)\right)$, where both $\psi(f)$ and $\eta_{1}(f)$ are $\Sigma_{1}^{0}$, where $\eta_{0}(f)$ is $\Pi_{1}^{0}$, and where $\forall f \in 2^{\mathbb{N}}\left(\psi(f) \leftrightarrow \neg \psi^{\prime}(f)\right)$ for some $\Sigma_{1}^{0}$ formula $\psi^{\prime}(f)$. By Lemma 2.13 , there are $\Pi_{0}^{0}$ formulae $\theta_{0}(x)$ and $\theta_{1}(x)$ such that $\psi(f) \leftrightarrow \exists n \theta_{0}(f[n])$ and $\neg \psi(f) \leftrightarrow \exists n \theta_{1}(f[n])$. By $\Delta_{1}^{0}$ comprehension, set a tree $T=\left\{s \in 2^{<\mathbb{N}}: \forall t \subseteq s\left(\neg \theta_{0}(t) \wedge \neg \theta_{1}(t)\right)\right\}$. $T$ has no infinite path, otherwise there exists an infinite sequence $f \in 2^{\mathbb{N}}$ such that $\forall n \neg \theta_{0}(f[n])$ and $\forall n \neg \theta_{1}(f[n])$, which is impossible. By weak König's lemma, $T$ is a finite tree. Let $n_{T}$ be the least even $n \in \mathbb{N}$ such that $|t|<n$ for all $t \in T$. Note that every $s \in 2^{<\mathbb{N}}$ of length $n_{T}$ satisfies exactly one of $\exists t \subseteq s \theta_{0}(t)$ and $\exists t \subseteq s \theta_{1}(t)$. By 3 and 4 of Remark 3.5, bounded $\Sigma_{1}^{0}$ comprehension, which is equivalent to $\Sigma_{1}^{0}$ induction, provides

$$
W_{i}=\left\{s \in 2^{\mathbb{N}}:|s|=n_{T} \wedge \exists t \subseteq s \theta_{i}(t) \wedge\left(\text { Player I wins } \eta_{i}(f) \text { at } s\right)\right\} \quad(i<2) .
$$

Then we can define a new $\Delta_{1}^{0}$ game $\varphi^{*}(f) \equiv f\left[n_{T}\right] \in W_{0} \cup W_{1}$. By $\Delta_{1}^{0}$-Det*, which is provided by $\mathrm{WKL}_{0}$, one of the players has a winning strategy in $\varphi^{*}(f)$. We show the following claim, which completes the proof of this theorem.

Claim The player who wins $\varphi^{*}(f)$ also wins the original game $\varphi(f)$.

Proof of the claim: First, assume that player I has a winning strategy $\sigma^{*}$ in $\varphi^{*}(f)$. He can win $\varphi(f)$ by playing as follows. He follows $\sigma$ till he reaches $s$ of length $n_{T}$. By the assumption, $s$ satisfies $s \in W_{0} \cup W_{1}$. If he reaches $s \in W_{i}$ then he switches the strategy to a winning strategy $\sigma_{s}$ in $\eta_{i}(s * f)$. More precisely, by 3 and 4 of Remark 3.5 and Lemma 3.6, we have a sequence $\left\langle\sigma_{s}: s \in W_{0} \cup W_{1}\right\rangle$ of strategies for player I such that $\sigma_{s}$ is a winning strategy in $\eta_{i}(s * f)$ for each $s \in W_{i}$. Now, a new strategy $\sigma$ for player I in $\varphi(f)$ is defined as follows:

$$
\sigma(t)= \begin{cases}\sigma_{t\left[n_{T}\right]}\left(t \ominus n_{T}\right) & \text { if }|t| \geq n_{T} \text { and } t\left[n_{T}\right] \in W_{0} \cup W_{1}, \\ \sigma^{*}(t) & \text { otherwise. }\end{cases}
$$

It can be checked that for any play $f$ in which player I follows $\sigma, f\left[n_{T}\right] \in W_{0} \cup W_{1}$ and $f \ominus n_{T}$ is a play in which player I follows $\sigma_{f\left[n_{T}\right]}$, and so $\eta_{i}(f)$ if $f\left[n_{T}\right] \in W_{i}$. Thus player I wins $\varphi(f)$.

Next, assume that player II has a winning strategy $\tau^{*}$ in $\varphi^{*}(f)$. She can win $\varphi(f)$ by playing as follows. She follows $\tau^{*}$ till she reaches $s$ of length $n_{T}$. By the assumption, $s$ is not in $W_{0} \cup W_{1}$. Note that $s$ satisfies exactly one of $\exists t \subseteq s \theta_{0}(t)$ and $\exists t \subseteq s \theta_{1}(t)$ and that if $\exists t \subseteq s \theta_{i}(t)$ and $s \notin W_{i}$, then player II wins $\eta_{i}(f)$ at $s$ by $\Sigma_{1}^{0}$-Det*. Then she switches the strategy to a winning strategy $\tau_{s}$ in $\eta_{i}(s * f)$, if $s$ satisfies $\exists t \subseteq s \theta_{i}(t)$. More precisely, we have a sequence $\left\langle\tau_{s}:|s|=n_{T} \wedge s \notin W_{0} \cup W_{1}\right\rangle$ of strategies for player II such that if $\exists t \subseteq s \theta_{i}(t)$, then $\tau_{s}$ is a winning strategy in $\eta_{i}(s * f)$ by Lemma 3.6 as in the previous case. Define a strategy $\tau$ for player II as follows:

$$
\tau(t)= \begin{cases}\tau_{t\left[n_{T}\right]}\left(t \ominus n_{T}\right) & \text { if }|t| \geq n_{T} \text { and } t\left[n_{T}\right] \notin W_{0} \cup W_{1}, \\ \tau(t) & \text { otherwise. }\end{cases}
$$

Then $\tau$ is a winning strategy for player II in $\varphi(f)$.

Finally, we have the following.

Corollary 3.8 RCA $\mathrm{R}_{0}^{*} \vdash \operatorname{Bisep}\left(\Delta_{1}^{0}, \Sigma_{1}^{0}\right)-$ Det $^{*} \leftrightarrow \mathrm{WKL}_{0}$. 


\section{$4 \quad \mathrm{ATR}_{0}+\Sigma_{1}^{1}$ induction and determinacy}

In this section, we consider the system $\mathrm{ATR}_{0}+\Sigma_{1}^{1}$ induction and determinacy in the Cantor space. First, the system $\mathrm{ATR}_{0}$ is defined as follows.

Definition 4.1 The system $\mathrm{ATR}_{0}$ consists of $\mathrm{RCA}_{0}$ plus the schema of $\Pi_{0}^{1}$ transfinite recursion.

Game theoretical characterizations of $\mathrm{ATR}_{0}$ are given as follows.

Proposition 4.2 RCA $\vdash \Delta_{1}^{0}$-Det $\leftrightarrow \Sigma_{1}^{0}$-Det $\leftrightarrow \Delta_{2}^{0}$-Det ${ }^{*} \leftrightarrow \Sigma_{2}^{0}$-Det ${ }^{*} \leftrightarrow \mathrm{ATR}_{0}$

Proof. For the equivalences between $\Delta_{1}^{0}$-Det, $\Sigma_{1}^{0}$-Det and $\Pi_{0}^{1}$ transfinite recursion, see [8, Section V.8] or [10]. For the equivalence between $\Delta_{2}^{0}$-Det ${ }^{*}, \Sigma_{2}^{0}$-Det ${ }^{*}$ and $\Pi_{0}^{1}$ transfinite recursion, see [5]. The base theory $\mathrm{RCA}_{0}$ can be replaced with $\mathrm{RCA}_{0}^{*}$ by Theorem 3.3.

Using the proposition, we can prove the following:

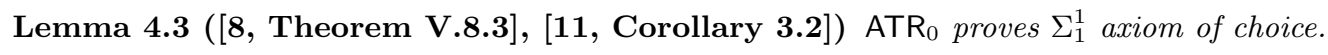

Remark 4.4 1. For any $\Pi_{2}^{0}$ game $\varphi(f)$ in the Cantor space, the assertion " $\sigma$ is a winning strategy for player I in $\varphi(f)$ " is equivalent to a $\Pi_{2}^{0}$ formula over $\mathrm{WKL}_{0}^{*}$. Let $\varphi(f) \equiv \forall n \exists m \theta(n, f[m])$. The assertion can be written as $\forall \tau \in \mathcal{S}_{\mathrm{II}}^{\{0,1\}} \forall n \exists m \theta(n,(\sigma \otimes \tau)[m]) \leftrightarrow \forall n \forall \tau \in \mathcal{S}_{\mathrm{II}}^{\{0,1\}} \exists m \theta(n,(\sigma \otimes \tau)[m])$. The underlined part is $\Sigma_{1}^{0}$ by Lemma 3.4 , and so the whole statement is $\Pi_{2}^{0}$.

2. For any $\Sigma_{2}^{0}$ game $\varphi(f)$ in the Cantor space, the assertion " $\sigma$ is a winning strategy for player I in $\varphi(f)$ " is equivalent to a $\Pi_{1}^{1}$ formula over $\mathrm{RCA}_{0}^{*}$. This is straightforward.

3. For any $\Pi_{2}^{0}$ game $\varphi(f)$ in the Cantor space, the assertion "player I has a winning strategy in $\varphi(f)$ " is equivalent to a $\Sigma_{1}^{1}$ formula over $W K L_{0}^{*}$. This follows from 1 .

4. For any $\Sigma_{2}^{0}$ game $\varphi(f)$ in the Cantor space, the assertion "player I has a winning strategy in $\varphi(f)$ " is equivalent to a $\Pi_{1}^{1}$ formula over $\mathrm{ATR}_{0}$. By a similar argument to 3 , the assertion "player II has a winning strategy in $\varphi(f)$ " can be written as a $\Sigma_{1}^{1}$ formula $\psi$. By $\Sigma_{2}^{0}$-Det ${ }^{*}$, which is provided by $A T R_{0}$, the aimed assertion is equivalent to $\neg \psi$ and is $\Pi_{1}^{1}$.

Now we consider the system $\mathrm{ATR}_{0}+\Sigma_{1}^{1}$ induction.

Remark 4.5 (1) [8, Corollary IX. 4. 7] shows that $\mathrm{ATR}_{0}+\Sigma_{1}^{1}$ induction proves the consistency of $A T R_{0}$. This means that $\Sigma_{1}^{1}$ induction really strengthens $A T R_{0}$.

(2) $\mathrm{ATR}_{0}+\Pi_{1}^{1}$ transfinite induction proves the consistency of $\mathrm{ATR}_{0}+\Sigma_{1}^{1}$ induction, since [8, Theorem VIII.3.15] shows that $\mathrm{ATR}_{0}$ can be axiomatized by $\mathrm{ACA}_{0}$ plus a single $\Pi_{2}^{1}$ formula and since [8, Theorem VIII.5.12] shows that $\mathrm{ATR}_{0}+\Pi_{1}^{1}$ transfinite induction implies the existence of a countable coded $\omega$-model of $\mathrm{ATR}_{0}$ and hence the consistency of $\mathrm{ATR}_{0}+$ full induction.

We have the following equivalence on $\Sigma_{1}^{1}$ induction.

Lemma 4.6 ([7, 2.1 Lemma]) $\Sigma_{1}^{1}$ induction is equivalent to bounded $\Sigma_{1}^{1}$ comprehension over $\mathrm{ACA}_{0}$, i.e., the system $\mathrm{RCA}_{0}$ plus $\Sigma_{1}^{0}$ comprehension.

[7] shows that $\Sigma_{1}^{1}$ induction is equivalent to $\Sigma_{1}^{1}$ transfinite induction over $\mathrm{ATR}_{0}$.

Although the intuitive idea of the following proof is the same as Theorem 3.7, the lack of $\Pi_{1}^{1}$ axiom of choice makes the proof slightly complicated.

Theorem 4.7 ATR $\mathrm{AT}_{0}+\Sigma_{1}^{1}$ induction proves $\operatorname{Bisep}\left(\Delta_{1}^{0}, \Sigma_{2}^{0}\right)$-Det* 
Proof. By Remark 2.16, it suffices to prove $\operatorname{Sep}\left(\Delta_{1}^{0}, \Sigma_{2}^{0}\right)$-Det* in $\operatorname{ATR}_{0}+\Sigma_{1}^{1}$ induction. Let $\varphi(f)$ be a game of the form $\left(\exists n \theta_{0}(f[n]) \wedge \eta_{0}(f)\right) \vee\left(\exists n \theta_{1}(f[n]) \wedge \eta_{1}(f)\right)$, where $\theta_{0}(s)$ and $\theta_{1}(s)$ are $\Pi_{0}^{0}$ such that $\forall f\left(\exists n \theta_{0}(f[n]) \leftrightarrow \neg \exists n \theta_{1}(f[n])\right)$, where $\eta_{0}(f)$ is $\Pi_{2}^{0}$, and where $\eta_{1}(f)$ is $\Sigma_{2}^{0}$. As in the proof of Theorem 3.7, define a tree $T=\left\{s \in 2^{<\mathbb{N}}: \forall t \subseteq s\left(\neg \theta_{0}(t) \wedge \neg \theta_{1}(t)\right)\right\}$ and take the least even $n_{T}$ with $|s|<n_{T}$ for all $s \in T$. Bounded $\Pi_{1}^{1}$ comprehension with help of 3 and 4 of Remark 4.4 yields

$$
\begin{aligned}
& W_{i}=\left\{s \in 2^{<\mathbb{N}}:|s|=n_{T} \wedge \exists t \subseteq s \theta_{i}(t) \wedge \text { player I wins } \eta_{i}(f) \text { at } s\right\} \\
& W_{i}^{\prime}=\left\{s \in 2^{<\mathbb{N}}:|s|=n_{T} \wedge \exists t \subseteq s \theta_{i}(t) \wedge s \notin W_{i}^{\prime}\right\}
\end{aligned}
$$

for $i<2$. Note that, by $\Sigma_{1}^{0}$-Det* ${ }^{*}$, player II wins $\eta_{i}(f)$ at $s \in W_{i}^{\prime}$.

Define a new $\Delta_{1}^{0}$ game $\varphi^{*}(f) \equiv f\left[n_{T}\right] \in W_{0} \cup W_{1}$. By $\Delta_{1}^{0}$-Det ${ }^{*}$, it is determinate.

Claim The player who wins $\varphi^{*}(f)$ also wins the original game $\varphi(f)$.

Proof of the claim. First, assume that player I has a winning strategy $\sigma^{*}$ in $\varphi^{*}(f)$. Then consider the following game: $\psi_{0}(f) \equiv\left(f\left[n_{T}\right] \in W_{0} \cup W_{1}\right) \wedge\left(f\left[n_{T}\right] \in W_{1} \rightarrow \eta_{1}(f)\right)$. Player II has no winning strategy in $\psi_{0}(f)$ as shown below. Suppose, for contradiction, player II has a winning strategy $\nu$ in $\psi_{0}(f)$. Then $\left(\sigma^{*} \otimes \nu\right)\left[n_{T}\right] \in W_{1}$, for otherwise, $\left(\sigma^{*} \otimes \nu\right)\left[n_{T}\right] \in W_{0}$ would hold and player II would lose $\psi_{0}(f)$. By the definition of $W_{1}$, player I has a winning strategy $v$ in $\eta_{1}\left(\left(\sigma^{*} \otimes \nu\right)\left[n_{T}\right] * f\right)$. Then define a strategy $\rho$ for player I in $\psi_{0}(f)$ as follows:

$$
\rho(t)= \begin{cases}\sigma^{*}(t) & \text { if }|u|<n_{T}, \\ v\left(t \ominus n_{T}\right) & \text { otherwise. }\end{cases}
$$

Since $(\rho \otimes \nu)\left[n_{T}\right]=\left(\sigma^{*} \otimes \nu\right)\left[n_{T}\right]$ and $(\rho \otimes \nu) \ominus n_{T}$ is a resulting play of $v, \eta_{1}(\rho \otimes \nu)$ holds, and so $\psi_{0}(\rho \otimes \nu)$ holds, which contradicts the assumption that $\nu$ is a winning strategy for player II in $\psi_{0}(f)$.

Since $\psi_{0}(f)$ is equivalent to some $\Sigma_{2}^{0}$ formula, player I has a winning strategy $\sigma_{0}$ in $\psi_{0}(f)$ by $\Sigma_{2}^{0}$-Det* ${ }^{*}$ Let $\left\langle\sigma_{s}: s \in W_{0}\right\rangle$ be a sequence of winning strategies for player I in $\eta_{0}(s * f)$, which is yielded by Lemma 4.3. Define a strategy $\sigma$ for player I in $\varphi(f)$ :

$$
\sigma(t)= \begin{cases}\sigma_{t\left[n_{T}\right]}\left(t \ominus n_{T}\right) & \text { if } t\left[n_{T}\right] \in W_{0} \\ \sigma_{0}(t) & \text { otherwise. }\end{cases}
$$

Then, for any strategy $\tau$ for player II in $\varphi(f),(\sigma \otimes \tau)\left[n_{T}\right] \in W_{0} \cup W_{1}$ holds and, for any $i<2$, if $(\sigma \otimes \tau)\left[n_{T}\right] \in W_{i}$, then $\eta_{i}(\sigma \otimes \tau)$ holds, and so $\varphi(\sigma \otimes \tau)$.

Next, assume that player II has a winning strategy $\tau^{*}$ in $\varphi^{*}(f)$. Note that $\varphi^{*}(f) \leftrightarrow \neg\left(f\left[n_{T}\right] \in\right.$ $\left.W_{0}^{\prime} \cup W_{1}^{\prime}\right)$. We can prove that player II has a winning strategy $\tau_{0}$ in $\psi_{1}(f) \equiv \neg\left(\left(f\left[n_{T}\right] \in W_{0}^{\prime} \cup W_{1}^{\prime}\right) \wedge\right.$ $\left.\left(f\left[n_{T}\right] \in W_{0}^{\prime} \rightarrow \neg \eta_{0}(f)\right)\right)$ as in the previous case. By Lemma 4.3, we have a sequence of winning strategies $\left\langle\tau_{s}:|s|=n_{T} \wedge \exists t \subseteq s \theta_{1}(t) \wedge s \notin W_{1}\right\rangle$ for player II in $\eta_{1}(s * f)$. Then the strategy $\tau$ for player II defined by

$$
\tau(t)= \begin{cases}\tau_{t\left[n_{T}\right]}\left(t \ominus n_{T}\right) & \text { if } t\left[n_{T}\right] \in W_{1}^{\prime}, \\ \tau_{0}(t) & \text { otherwise. }\end{cases}
$$

is a winning strategy for player II in the original game $\varphi(f)$.

Lemma 4.8 (normal form theorem 2, [8, Theorem V.1.4]) For a $\Sigma_{1}^{1}$ formula $\varphi(X)$, we can find a $\Pi_{0}^{0}$ formula $\theta(X, f)$ such that $\mathrm{ACA}_{0}$ proves $\forall Y\left(\varphi(Y) \leftrightarrow \exists g \in \mathbb{N}^{\mathbb{N}} \forall m \theta(Y[m], g[m])\right)$.

To investigate the other direction, some definitions and notations are needed.

Definition 4.9 A finite sequence $s \in 2^{<\mathbb{N}}$ is regular if: 
(r1) $s=\langle\rangle$ or $|s|>1 \wedge s(|s|-1) \neq s(|s|-2)$,

(r2) for all $i<2,2 k+i<|s|-1$ and $s(2 k+i)=i$ imply $s(2 k+i+1)=i$.

If $s \in 2^{<\mathbb{N}}$ is a non-empty regular sequence, $\bar{s}=\left\langle n_{0}, \ldots, n_{k}\right\rangle \in \mathbb{N}^{<\mathbb{N}}$ is the sequence such that

$$
s=\langle\underbrace{0, \ldots, 0}_{2 n_{0} \text { times }}, 1, \underbrace{1, \ldots, 1}_{2 n_{1} \text { times }}, 0, \ldots \underbrace{i_{k}, \ldots, i_{k}}_{2 n_{k} \text { times }}, 1-i_{k}\rangle
$$

where $i_{k}$ is 0 if $k$ is even, $i_{k}=1$ otherwise. The assertion " $s$ is regular" is equivalent to a $\Pi_{0}^{0}$ formula over RCA . $_{0}^{*}$

An infinite sequence $f \in 2^{\mathbb{N}}$ is totally regular if it satisfies

(r2') for all $i<2$ and $k, s(2 k+i)=i$ implies $s(2 k+i+1)=i$,

(r3) for all $i<2$ and $k$, if $f(k)=i$, then there exists $m>k$ with $f(m)=1-i$.

For a totally regular sequence $g \in 2^{\mathbb{N}}, \bar{g}=\left\langle n_{0}, n_{1}, n_{2}, \ldots\right\rangle \in \mathbb{N}^{\mathbb{N}}$ is the sequence such that

$$
g=\langle\underbrace{0, \ldots, 0}_{2 n_{0} \text { times }}, 1, \underbrace{1, \ldots, 1}_{2 n_{1} \text { times }}, 0, \underbrace{0, \ldots, 0}_{2 n_{2} \text { times }}, 1, \ldots\rangle .
$$

The assertion " $f$ is totally regular" is equivalent to a $\Pi_{2}^{0}$ formula over $\mathrm{RCA}_{0}^{*}$.

Theorem 4.10 $\mathrm{RCA}_{0}^{*}$ proves that $\operatorname{Bisep}\left(\Delta_{1}^{0}, \Sigma_{2}^{0}\right)$-Det* implies $\Sigma_{1}^{1}$ induction.

Proof. Assume that $\operatorname{Bisep}\left(\Delta_{1}^{0}, \Sigma_{2}^{0}\right)$-Det*. By Lemma 4.6, it suffices to prove bounded $\Sigma_{1}^{1}$ comprehension. Let $\varphi(x)$ be a $\Sigma_{1}^{1}$ formula. We can find a $\Pi_{0}^{0}$ formula $\theta(x, s)$ such that $\mathrm{ACA}_{0}$ proves $\varphi(x) \leftrightarrow \exists f \forall m \theta(x, f[m])$ for all $x \in \mathbb{N}$.

For any fixed $n$, consider the following game. First, player I chooses $k<n$ and asks whether $\varphi(k)$ or not. Then player II answers "Yes" or "No." If she answers "Yes," she has to construct a witness $g$ of $\varphi(k)$. If she answers "No," player I has to construct a witness of $\varphi(k)$. Such a game is realized as follows:

- Player I chooses $k<n$ by playing 0 at his first $k$-th turns and 1 at his $(k+1)$-th turn.

- Player II chooses 0 or 1 at her $(k+1)$-th turn.

- If player II answers 1 at her $(k+1)$-th turn, she wins by constructing $g$ such that $\forall m \theta(k, \bar{g}[m]))$. (case 1)

- If player II chooses 0 at her $(k+1)$-th turn, player I wins by constructing $g$ such that $\forall m \theta(k, \bar{g}[m])$. (case 2)

To illustrate, the game goes as follows:

Case 1

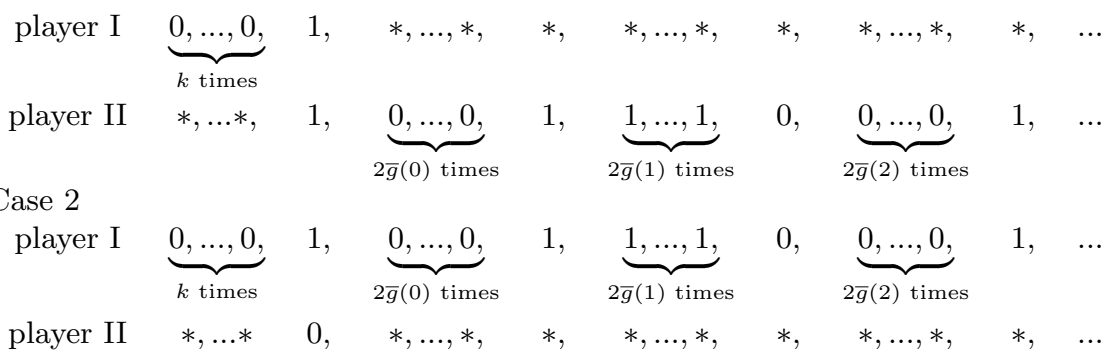

Player I wins if and only if one of the following holds:

- Player I chooses $k<n$ and player II answers "Yes" and player II fails to construct totally regular $g \in 2^{\mathbb{N}}$ with $\forall m \theta(k, \bar{g}[m])$. 
- Player I chooses $k<n$ and player II answers "No" and player I successes to construct totally regular $g \in 2^{\mathbb{N}}$ with $\forall m \theta(k, \bar{g}[m])$.

Formally, this winning condition can be written as below:

$$
\begin{gathered}
\exists k<l\left(\psi(f, k, 1) \wedge\left(\left(f_{k, 1} \text { is not totally regular }\right) \vee \exists l\left(\left(f_{k, 1}[l] \text { is regular }\right) \wedge \neg \theta\left(k, \overline{f_{k, 1}[l]}\right)\right)\right)\right) \vee \\
\exists k<l\left(\psi(f, k, 0) \wedge\left(f_{k, 0} \text { is totally regular }\right) \wedge \forall l \exists m\left(\left|\overline{f_{k, 0}[m]}\right|=l \wedge \theta\left(k, \overline{f_{k, 0}[m]}\right)\right)\right),
\end{gathered}
$$

where $\psi(f, k, i)$ is the $\Pi_{0}^{0}$ formula $\left.\forall i<k(f(2 i)=0) \wedge f(2 k)=1 \wedge f(2 k+1)=i\right)$ and $f_{k, i}$ is $g \in 2^{\mathbb{N}}$ such that, for all $l, g(l)=f(2(k+1)+2 l+i)$.

Similarly to the proof of Theorem 3.7, $\operatorname{Bisep}\left(\Delta_{1}^{0}, \Sigma_{2}^{0}\right)$-Det* implies that $(\sharp)$ is determinate. Moreover, player I cannot have a winning strategy in $(\sharp)$, since if $\varphi(k)$ holds, player I cannot win when player II answers "Yes" and constructs a witness, otherwise, i.e., $\varphi(k)$ holds, player I cannot win when player II answers "No." Therefore player II has a winning strategy $\tau . \Delta_{1}^{0}$ comprehension yields $Y=\left\{k<n: \tau\left(k^{\tau} *\langle 1\rangle\right)=1\right\}$. Clearly, $Y$ enjoys the desired property.

Since $\operatorname{Bisep}\left(\Delta_{1}^{0}, \Sigma_{2}^{0}\right)$-Det* implies $\Sigma_{2}^{0}$-Det ${ }^{*}$, it implies $\Pi_{0}^{1}$ transfinite recursion by Proposition 4.2 . Finally, we have the following.

Corollary 4.11 $\mathrm{RCA}_{0}^{*} \vdash \operatorname{Bisep}\left(\Delta_{1}^{0}, \Sigma_{2}^{0}\right)$-Det ${ }^{*} \leftrightarrow \mathrm{ATR}_{0}+\Sigma_{1}^{1}$ induction.

\section{$5 \quad \Pi_{1}^{1}-\mathrm{CA}_{0}$ and the determinacy of Wadge class}

In this section, we find determinacy schemata which is equivalent to $\Pi_{1}^{1}$ comprehension.

Definition 5.1 $\Pi_{1}^{1}-\mathrm{CA}_{0}$ is the system $\mathrm{RCA}$ plus $\Pi_{1}^{1}$ comprehension.

By [8, Theorem V.5.1], $\Pi_{1}^{1}-\mathrm{CA}_{0}$ includes $A T R_{0}$. Moreover, since $\Pi_{1}^{1}-C A_{0}$ proves both $\Pi_{1}^{1}$ and $\Sigma_{1}^{1}$ transfinite induction by Lemma 2.3, $\Pi_{1}^{1}-\mathrm{CA}_{0}$ includes $\mathrm{ATR}_{0}+\Sigma_{1}^{1}$ induction and, by Remark 4.5 (2), proves the consistency of $\mathrm{ATR}_{0}+\Sigma_{1}^{1}$ induction.

By determinacies in the Baire space, $\Pi_{1}^{1}-\mathrm{CA}_{0}$ is characterized as follows:

Proposition 5.2 RCA $\mathrm{RC}_{0}^{*} \vdash \operatorname{Bisep}\left(\Delta_{1}^{0}, \Sigma_{1}^{0}\right)$-Det $\leftrightarrow\left(\Sigma_{1}^{0}\right)_{2}$-Det $\leftrightarrow \Pi_{1}^{1}-\mathrm{CA}_{0}$, where $\left(\Sigma_{1}^{0}\right)_{2}$ is $\Sigma_{1}^{0} \wedge \Pi_{1}^{0}$.

Proof. See [8, Lemma VI.5.2, Lemma VI.5.3]. Although the proof of [8, Lemma VI.5.3] shows the implication from $\left(\Sigma_{1}^{0}\right)_{2}$-Det to $\Pi_{1}^{1}$ comprehension in $\operatorname{RCA}$, Bisep $\left(\Delta_{1}^{0}, \Sigma_{1}^{0}\right)$-Det implies the determinacy of the game defined in the proof and so we need only $\operatorname{Bisep}\left(\Delta_{1}^{0}, \Sigma_{1}^{0}\right)$-Det for the proof.

By the above proposition, it turns out that there is no determinacy schemata corresponding to Wadge classes which are equivalent to $\mathrm{ATR}_{0}+\Sigma_{1}^{1}$ induction.

In the following proof, we construct a game which is similar to that in the proof of Theorem 4.10. In the following game player I can ask about any natural number $k$, whereas he can ask only about $k<n$ in the game defined in the proof of Theorem 4.10. This point corresponds to the difference between (unbounded) $\Sigma_{1}^{1}$ comprehension and bounded $\Sigma_{1}^{1}$ comprehension.

Theorem 5.3 $\mathrm{RCA}_{0}^{*}$ proves that $\operatorname{Bisep}\left(\Sigma_{1}^{0}, \Sigma_{2}^{0}\right)$-Det* implies $\Pi_{1}^{1}$ comprehension.

Proof. Assume $\operatorname{Bisep}\left(\Sigma_{1}^{0}, \Sigma_{2}^{0}\right)$-Det*. Since we can easily see $\operatorname{Bisep}\left(\Sigma_{1}^{0}, \Sigma_{2}^{0}\right)$-Det* implies $\Sigma_{1}^{0} \wedge \Pi_{1}^{0}$ Det*, we can work in $\mathrm{ACA}_{0}$. We show $\Sigma_{1}^{1}$ comprehension, which is equivalent to $\Pi_{1}^{1}$ comprehension over $\mathrm{RCA}_{0}^{*}$. Let $\varphi(n)$ be a $\Sigma_{1}^{1}$ formula. In $\mathrm{ACA}_{0}$, we can find a $\Pi_{0}^{0}$ formula $\theta(x, f)$ such that $\varphi(n) \leftrightarrow \exists f \forall m \theta(n, f[m])$, for all $n \in \mathbb{N}$. Now we consider the following game: Player I chooses $k \in \mathbb{N}$ and asks whether $\varphi(n)$ or not. Player II answers "Yes" or "No." If she answers "Yes," she has to construct a witness for $\varphi(n)$. If she answers "No," player I has to construct a witness for $\varphi(n)$.

Such a game is realized as follows: 
- Player I chooses $k \in \mathbb{N}$ by playing 0 his first $k$ turns and 1 at his $(k+1)$-th turn.

- Player II chooses 0 or 1 at her $(k+1)$-th turn.

- If player II chooses 1 , she wins by constructing totally regular $g \in 2^{\mathbb{N}}$ with $\forall m \theta(n, \bar{g}[m])$.

- If player II chooses 0 , player I wins by constructing totally regular $g \in 2^{\mathbb{N}}$ with $\forall m \theta(n, \bar{g}[m])$.

Player I wins if and only if one of the following conditions holds:

- Player I chooses $k$ and player II answers "Yes," and she fails to construct $g$ with $\forall m \theta(k, \bar{g}[m])$.

- Player I chooses $k$ and player II answers "No," and he successes to construct $g$ with $\forall m \theta(k, \bar{g}[m])$.

Rigorously, the above winning condition can be written as below:

$$
\begin{gathered}
\exists k\left(\psi(f, k, 1) \wedge\left(\left(f_{k, 1} \text { is not totally regular }\right) \vee \exists l\left(\left(f_{k, 1}[l] \text { is regular }\right) \wedge \neg \theta\left(k, \overline{f_{k, 1}[l]}\right)\right)\right)\right) \vee \\
\exists k\left(\psi(f, k, 0) \wedge\left(\left(f_{k, 0} \text { is totally regular }\right) \wedge \forall l \exists m\left(\left|\overline{f_{k, 0}[m]}\right|=l \wedge \theta\left(k, \overline{f_{k, 0}[m]}\right)\right)\right)\right),
\end{gathered}
$$

where $\psi(f, k, i)$ is the $\Pi_{0}^{0}$ formula $\left.\forall i<k(f(2 i)=0) \wedge f(2 k)=1 \wedge f(2 k+1)=i\right)$ and $f_{k, i}$ is $g \in 2^{\mathbb{N}}$ such that, for all $l, g(l)=f(2(k+1)+2 l+i)$. As in the proof of Theorem 4.10, Bisep $\left(\Sigma_{1}^{0}, \Sigma_{2}^{0}\right)$-Det* implies that player II has a winning strategy $\tau$ in (q). Then $Y=\left\{k: \tau\left(k^{\tau} *\langle 1\rangle\right)=1\right\}$ enjoys the desired property.

Next, we consider the converse. It suffices to show the implication from $\Pi_{1}^{1}-\mathrm{CA}_{0}$ to $\operatorname{Sep}\left(\Sigma_{1}^{0}, \Sigma_{2}^{0}\right)$ Det* ${ }^{*}$ since it is easy to prove that $\operatorname{Sep}\left(\Sigma_{1}^{0}, \Sigma_{2}^{0}\right)$-Det* implies $\operatorname{Bisep}\left(\Sigma_{1}^{0}, \Sigma_{2}^{0}\right)$-Det ${ }^{*}$. It can be proved in a similar way to Theorem 4.7. As mentioned before Theorem 4.7, if we had a sufficient fragment of the axiom of choice, it could be proved in a simpler way like Theorem 3.7. Now, although $\Pi_{1}^{1}-\mathrm{CA}_{0}$ does not prove $\Pi_{1}^{1}$ axiom of choice, we have the following conservation results.

Lemma 5.4 ([8, Theorem IX.4.9]) $\Pi_{1}^{1}-\mathrm{AC}_{0}$, the system $\mathrm{ACA}_{0}$ plus $\Pi_{1}^{1}$ axiom of choice, is conservative over $\Pi_{1}^{1}-\mathrm{CA}_{0}$ for $\Pi_{3}^{1}$ sentence, i.e., any $\Pi_{3}^{1}$ sentence $\psi$ which is provable in $\Pi_{1}^{1}-\mathrm{AC}_{0}$ is already provable in $\Pi_{1}^{1}-\mathrm{CA}_{0}$.

Since all the sentences in $\operatorname{Sep}\left(\Sigma_{1}^{0}, \Sigma_{2}^{0}\right)$-Det* are equivalent to $\Pi_{3}^{1}$ formulae, Lemma 5.4 guarantees that if we can prove $\operatorname{Sep}\left(\Sigma_{1}^{0}, \Sigma_{2}^{0}\right)$-Det ${ }^{*}$ in $\Pi_{1}^{1}-\mathrm{AC}_{0}$, then it is already provable in $\Pi_{1}^{1}-\mathrm{CA}_{0}$,

Theorem 5.5 $\Pi_{1}^{1}-\mathrm{CA}_{0}$ proves $\operatorname{Sep}\left(\Sigma_{1}^{0}, \Sigma_{2}^{0}\right)$-Det*

Proof. As mentioned above, it suffices to prove $\operatorname{Sep}\left(\Sigma_{1}^{0}, \Sigma_{2}^{0}\right)$-Det* in $\Pi_{1}^{1}-\mathrm{AC}_{0}$. Let $\varphi(f)$ be a $\operatorname{Sep}\left(\Sigma_{1}^{0}, \Sigma_{2}^{0}\right)$ game, say $(\exists n \theta(f[n]) \wedge \psi(f)) \vee(\neg \exists n \theta(f[n]) \wedge \eta(f))$, where $\theta$ is $\Pi_{0}^{0}, \psi(f)$ is $\Pi_{2}^{0}$ and $\eta(f)$ is $\Sigma_{2}^{0}$. By 3 of Remark 4.4, $\Pi_{1}^{1}$ comprehension implies the existence of $V$ and $V^{\prime}$ such that

$$
\begin{aligned}
V & =\{s:|s| \text { is even, } \exists t \subseteq s \theta(t) \text { and player I wins } \psi(f) \text { at } s\}, \\
V^{\prime} & =\{s:|s| \text { is even, } \exists t \subseteq s \theta(t) \text { and } s \notin V\} .
\end{aligned}
$$

Define a new game $\varphi^{*}(f)$ as follows: $\varphi^{*}(f) \equiv \exists n(f[n] \in V) \vee(\neg \exists n \theta(f[n]) \wedge \eta(f))$. By $\Sigma_{2}^{0}$-Det*, which is proved in $\mathrm{ATR}_{0}$ (cf. Proposition 4.2), $\varphi^{*}(f)$ is determinate. We show the following claim:

Claim The player who wins $\varphi^{*}(f)$ also wins in the original game $\varphi(f)$.

Proof of the claim First, assume that player I has a winning strategy $\sigma^{*}$ in $\varphi^{*}(f)$. By $\Sigma_{1}^{1}$ axiom of choice, which is proved in $\mathrm{ATR}_{0}$, we have a sequence $\left\langle\sigma_{s}: s \in V\right\rangle$ of winning strategies for player I in $\psi(s * f)$. Now we define a new strategy $\sigma$ for player I in $\varphi(f)$ as follows:

$$
\sigma(s)= \begin{cases}\sigma^{*}(s) & \text { if } t \notin V \text { for all } t \subseteq s, \\ \sigma_{t}(s \ominus|t|) & \text { if } t \text { is the } \subseteq \text {-least initial segment of } s \text { with } t \in V .\end{cases}
$$


We prove that $\sigma$ is a winning strategy for player $\mathrm{I}$ in $\varphi(f)$. Let $f$ be a play in which player I follows $\sigma$. If there is no $k \in \mathbb{N}$ with $\theta(f[k])$, then $f$ satisfies $\neg \exists n \theta(f[n]) \wedge \eta(f)$, and so player I wins. If there is $k$ with $\theta(f[k])$, then $f$ satisfies $\exists m f[2 m] \in V$. Take the least such $m$. Then $\sigma(f[2 m+2 l])=\sigma_{f[2 m]}(f[2 m+2 l] \ominus 2 m)$ holds for all $l$, and so $\psi(f)$ holds, and so player I wins.

Next, assume that player II has a winning strategy $\tau^{*}$ in the game $\varphi^{*}(f)$. Since ATR implies $_{0}$ $\Sigma_{2}^{0}$-Det ${ }^{*}$, player II wins $\psi(f)$ at each $s \in V^{\prime}$. By $\Pi_{1}^{1}$ axiom of choice, we have a sequence $\left\langle\tau_{s}: s \in V^{\prime}\right\rangle$ of winning strategies for player II in $\psi(s * f)$.

Now, define a new strategy $\tau$ for player II in $\varphi(f)$ as follows:

$$
\tau(s)= \begin{cases}\tau^{*}(s) & \text { if } t \notin V^{\prime} \text { for all } t \subseteq s, \\ \tau_{t}(s \ominus|t|) & \text { if } t \text { is the } \subseteq \text {-least initial segment of } s \text { with } t \in V^{\prime} .\end{cases}
$$

We can prove that $\tau$ is a winning strategy for player II in $\varphi(f)$, similarly to the previous case.

Corollary 5.6 RCA RC $_{0}^{*} \operatorname{Bisep}\left(\Sigma_{1}^{0}, \Sigma_{2}^{0}\right)-\operatorname{Det}^{*} \leftrightarrow \operatorname{Sep}\left(\Sigma_{1}^{0}, \Sigma_{2}^{0}\right)-$ Det $^{*} \leftrightarrow \Pi_{1}^{1}-\mathrm{CA}_{0}$.

Proof. Theorem 5.3 shows the implication of $\Pi_{1}^{1}$ comprehension from $\operatorname{Bisep}\left(\Sigma_{1}^{0}, \Sigma_{2}^{0}\right)$-Det* . Theorem 5.5 shows the implication from $\Pi_{1}^{1}$ comprehension to $\operatorname{Sep}\left(\Sigma_{1}^{0}, \Sigma_{2}^{0}\right)$-Det* . The implication from $\operatorname{Sep}\left(\Sigma_{1}^{0}, \Sigma_{2}^{0}\right)$-Det* to $\operatorname{Bisep}\left(\Sigma_{1}^{0}, \Sigma_{2}^{0}\right)$-Det* is clear.

\section{$6 \Pi_{1}^{1}-\mathrm{TR}_{0}$ and determinacy of Wadge class}

Now let us turn to considering the relation between $\Pi_{1}^{1}$ transfinite recursion and determinacy.

Definition 6.1 $\Pi_{1}^{1}-\mathrm{TR}_{0}$ is the system RCA plus $\Pi_{1}^{1}$ transfinite recursion.

$\Pi_{1}^{1}-\mathrm{TR}_{0}$ is characterized by determinacy as follows:

Proposition 6.2 ([11, Theorem 6.1]) $\mathrm{RCA}_{0}^{*} \vdash \Delta_{2}^{0}$-Det $\leftrightarrow \Pi_{1}^{1}-\mathrm{TR}_{0}$.

In this section, we prove the equivalence between $\operatorname{Sep}\left(\Delta_{2}^{0}, \Sigma_{2}^{0}\right)$-Det* and $\Pi_{1}^{1}-\mathrm{TR}_{0}$ over $\mathrm{RCA}$.

Theorem 6.3 $\mathrm{RCA}_{0}^{*}$ proves that $\operatorname{Sep}\left(\Delta_{2}^{0}, \Sigma_{2}^{0}\right)$-Det* implies $\Delta_{2}^{0}$-Det.

Proof. We work in $\operatorname{RCA}_{0}^{*}$. Let $\varphi(f)$ be a game in the Baire space such that $\forall f \in \mathbb{N}^{\mathbb{N}}((\varphi(f) \leftrightarrow$ $\left.\forall n \exists m \theta_{0}(n, f[m])\right)$ and $\left.\forall f \in \mathbb{N}^{\mathbb{N}}\left(\neg \varphi(f) \leftrightarrow \forall n \exists m \theta_{1}(n, f[m])\right)\right)$ for some $\Pi_{0}^{0}$ formulae $\theta_{0}(x, s)$ and $\theta_{1}(x, s)$.

We shall define a game $\varphi^{*}(f)$ in the Cantor space such that the player who wins $\varphi^{*}(f)$ also wins $\varphi(f)$ and $\operatorname{Sep}\left(\Delta_{2}^{0}, \Sigma_{2}^{0}\right)$-Det ${ }^{*}$ proves its determinacy. The intuitive idea of $\varphi^{*}(f)$ is as follows. Instead of playing in the Baire space, players I and II construct a totally regular sequence $f \in 2^{\mathbb{N}}$ (recall Definition 4.9). The first player who gives up making $f$ totally regular loses. If they succeed to construct totally regular $f$, then player I wins if and only if $\varphi(\bar{f})$. To give a rigorous formulation to this idea, first let

$$
\begin{aligned}
& \psi_{0}(k, i, f) \equiv f(2 k+i)=i \rightarrow f(2 k+i+1)=i, \\
& \psi_{1}(i, f) \equiv \exists n\left(\forall k<n \forall j<2 \psi_{0}(k, j, f) \wedge \forall l<(1-i) \psi_{0}(n, l, f) \wedge \neg \psi_{0}(n, 1-i, f)\right),
\end{aligned}
$$

(This means "player I (resp. II) first breaks (r2') of Definition 4.9" if $i=0$ (resp. $i=1)$ )

$\psi_{2}(i, f) \equiv \forall n \forall i<2 \psi_{0}(n, j, f) \wedge \exists m \forall l>m(f(2 l+i)=i)$,

(This means "both players keep (r2') but player I (resp. II) breaks (r3)" if $i=0$ (resp. $i=1)$.) $\psi(i, f) \equiv \psi_{1}(i, f) \vee \psi_{2}(i, f)$.

(This means "player I (resp. II) first gives up making $f$ totally regular" if $i=0$ (resp. $i=1)$.) 
Note that $\psi(i, f)$ is equivalent to a $\Sigma_{2}^{0}$ formula over $\mathrm{RCA}_{0}^{*}$. Then, for $i<2$, set $\eta_{i}(f)$ by ( $f$ is totally regular $) \wedge \forall n \exists m\left(f[m]\right.$ is regular $\left.\wedge \theta_{i}(n, \overline{f[m]})\right)$. Clearly $\eta_{i}$ is equivalent to a $\Pi_{2}^{0}$ formula over $\mathrm{RCA}_{0}^{*}$. Then there is no $f \in 2^{\mathbb{N}}$ with $\eta_{0}(f) \wedge \eta_{1}(f)$. By Lemma 2.17, $\operatorname{RCA}_{0}^{*}$ yields a $\Sigma_{2}^{0}$ formula $\eta(f)$ and a $\Pi_{2}^{0}$ formula $\eta^{\prime}(f)$ with $\forall f \in 2^{\mathbb{N}}\left(\left(\eta(f) \leftrightarrow \eta^{\prime}(f)\right) \wedge\left(\eta_{0}(f) \rightarrow \eta(f)\right) \wedge\left(\eta_{1}(f) \rightarrow \neg \eta(f)\right)\right)$. Furthermore, we can check each $f$ satisfies exactly one of $\psi(0, f), \psi(1, f)$ and $\eta_{i}(f)$ for each $f \in 2^{\mathbb{N}}$.

\begin{tabular}{|l|l|}
\hline \multicolumn{1}{|c|}{$\eta(f)$} & $\neg \eta(f)$ \\
\hline$\psi(0, f)$ & $\psi(0, f)$ \\
\hline$\eta_{0}(f)$ & $\eta_{1}(f)$ \\
\hline$\psi(1, f)$ & $\psi(1, f)$ \\
\hline
\end{tabular}

Define a new game $\varphi^{*}(f)$ by $(\eta(f) \wedge \neg \psi(0, f)) \vee(\neg \eta(f) \wedge \psi(1, f))$. $\operatorname{Sep}\left(\Delta_{2}^{0}, \Sigma_{2}^{0}\right)$-Det* implies that $\varphi^{*}(f)$ is determinate. Since $\varphi^{*}(f)$ holds if and only if $\eta_{0}(f) \vee \psi_{1}(1, f)$, we can check that player who has a winning strategy in $\varphi^{*}(f)$ also has a winning strategy in $\varphi(f)$.

For the converse, we need some preparations motivated by the reformulation of $\Delta_{2}^{0}$ in [11].

Lemma 6.4 For any $\Pi_{2}^{0}$ formula $\varphi(f)$ with a distinguished function variable $f \in 2^{\mathbb{N}}$, we can find, in $\mathrm{RCA}_{0}, a \Pi_{0}^{0}$ formula $\theta^{\prime}(x)$ in which $n$ does not occur such that $\forall f \in 2^{\mathbb{N}}\left(\varphi(f) \leftrightarrow \forall n \exists m>n \theta^{\prime}(f[m])\right)$.

Proof. See [3, Lemma 3.1]. Although the original proof is done in ACA $_{0}$, it works also in RCA.$\square$

Let $\psi(f)$ be a $\Sigma_{2}^{0}$ formula and $\psi^{\prime}(f) \Pi_{2}^{0}$ a formula such that $\forall f\left(\psi(f) \leftrightarrow \psi^{\prime}(f)\right)$. Then, by Lemma 6.4 , we can find $\Pi_{0}^{0}$ formulae $\theta_{0}(x)$ and $\theta_{1}(x)$ such that

$$
\psi(f) \leftrightarrow \forall n \exists m>n \theta_{0}(f[m]), \quad \neg \psi(f) \leftrightarrow \forall n \exists m>n \theta_{1}(f[m]) .
$$

We may assume that $\left\{s: \theta_{0}(s) \wedge \theta_{1}(s)\right\}=\emptyset$ and $\theta_{0}(\langle\rangle)$ by replacing $\theta_{0}(x)$ with $\theta_{0}^{\prime}(x) \equiv\left(\theta_{0}(x) \wedge\right.$ $\left.\neg \theta_{1}(x)\right) \vee(x=\langle\rangle)$ and $\theta_{1}(x)$ with $\theta_{1}^{\prime}(x) \equiv \theta_{1}(x) \wedge \neg \theta_{0}(x) \wedge(x \neq\langle\rangle)$ if necessary. Define a $T_{\theta_{0}, \theta_{1}}$ by

$$
\begin{aligned}
T_{\theta_{0}, \theta_{1}}=\left\{x \in\left(2^{<\mathbb{N}}\right)^{<\mathbb{N}}:\right. & x(0) \subsetneq x(1) \subsetneq \ldots \subsetneq x(|x|-1), \\
& \left.\theta_{0}(x(k)) \text { for each even } k<|x| \text { and } \theta_{1}(x(k)) \text { for each odd } k<|x|\right\}
\end{aligned}
$$

For every $f \in 2^{\mathbb{N}}$, we can prove that there exists $x \in T$ such that $x$ has no proper extension in $T$ and $\bigcup_{i<|x|} x(i)=x(|x|-1) \subseteq f$ as follows. If $\forall n \exists m>n \theta_{i}(f[m])$, there exists $p$ and $r>p$ with $\forall q>p \neg \theta_{1-i}(f[q])$ and $\theta_{i}(f[r])$. If $i=0,\langle f[r]\rangle$ enjoys the desired property. If $i=1,\langle\langle\rangle, f[r]\rangle$ enjoys the desired property.

$T_{\theta_{0}, \theta_{1}}$ has no infinite path, since if $F$ were an infinite path of $T_{\theta_{0}, \theta_{1}}, f \in 2^{\mathbb{N}}$ defined by $f(n)=$ $\bigcup_{n \in \mathbb{N}} F(n)=(F(n))(n)$ would satisfy both $\psi(f)$ and $\neg \psi(f)$. The restriction $\prec_{\theta_{0}, \theta_{1}}$ of Kleene Brouwer ordering to $T_{\theta_{0}, \theta_{1}}$ is defined as follows: For all $x, y \in T_{\theta_{0}, \theta_{1}}$,

$$
x \prec \theta_{\theta_{0}, \theta_{1}} y \quad \leftrightarrow \quad x \subseteq y \vee \exists j<\min (|x|,|y|)(x(j)<y(j) \wedge \forall i<j(x(i)=y(i))) .
$$

Then $\mathrm{ACA}_{0}$ proves that $\prec_{\theta_{0}, \theta_{1}}$ is a well ordering (cf. [8, Lemma V.1.3]).

Theorem 6.5 $\Pi_{1}^{1}-\mathrm{TR}_{0}$ proves $\operatorname{Sep}\left(\Delta_{2}^{0}, \Sigma_{2}^{0}\right)$-Det*

Proof. Let $\varphi(f)$ be a game in the Cantor space such that $\forall f \in 2^{\mathbb{N}}\left(\varphi(f) \leftrightarrow\left(\psi(f) \wedge \eta_{0}(f)\right) \vee(\neg \psi(f) \wedge\right.$ $\left.\left.\eta_{1}(f)\right)\right)$ for some $\Sigma_{2}^{0}$ formulae $\psi(f)$ and $\eta_{1}(f)$ and $\Pi_{2}^{0}$ formula $\eta_{0}(f)$. Assume that $\forall f\left(\psi(f) \leftrightarrow \psi^{\prime}(f)\right)$ for some $\Pi_{2}^{0}$ formula $\psi^{\prime}(f)$. Then we can find $\Pi_{0}^{0}$ formula $\theta_{0}$ and $\theta_{1}$ such that $\forall f \in 2^{\mathbb{N}}((\psi(f) \leftrightarrow$ $\left.\left.\forall n \exists m>n \theta_{0}(f[m])\right) \wedge\left(\neg \psi(f) \leftrightarrow \forall n \exists m>n \theta_{1}(f[m])\right)\right)$. Then take $T_{\theta_{0}, \theta_{1}}$ as mentioned above and define $T_{0}$ and $T_{1}$ as follows:

$$
T_{i}=\left\{x \in T_{\theta_{0}, \theta_{1}}: x \text { is has no proper extension in } T_{\theta_{0}, \theta_{1}} \text { and } \theta_{i}(x(|x|-1))\right\} .
$$


Then, for each $i<2$, the restriction $\prec_{i}$ of $\prec_{\theta_{0}, \theta_{1}}$ to $T_{i}$ is also well orderings. Note that, for every $f \in 2^{\mathbb{N}}$, there is $x \in T_{0} \cup T_{1}$ such that $x(|x|-1) \subseteq f$. For any $x \in T_{i}, \Pi_{1}^{1}$ transfinite recursion yields

$$
W_{x}^{i}=\left\{s \in 2^{<\mathbb{N}}: x(|x|-1) \subseteq s \wedge(|s| \text { is even }) \wedge\left(\text { player I wins }\left(\eta_{i}(f) \vee \exists n\left(f[n] \in \bigcup_{y \prec_{i} x} W_{y}^{i}\right)\right) \text { at } s\right)\right\} .
$$

Claim 1 Let $x \in T_{i}$. Then, for every $f \in 2^{\mathbb{N}}, \forall n \exists m>n \theta_{i}((x(|x|-1) * f)[m])$.

Proof of Claim 1 Let $x \in T_{i}$. We prove that, for any $s \in 2^{<\mathbb{N}}, \neg \theta_{1-i}(x(|x|-1) * s)$, which implies for any $f \in 2^{\mathbb{N}}, \neg \forall n \exists m>n \theta_{1-i}((x(|x|-1) * f)[m])$ and so $\forall n \exists m>n \theta_{0}((x(|x|-1) * f)[m])$. Suppose that $\theta_{1-i}(x(|x|-1) * s)$ for some $s \in 2^{<\mathbb{N}}$. Then $x *\langle s\rangle \in T_{\theta_{0}, \theta_{1}}$, which contradicts to the fact that $x$ has no proper extension in $T_{\theta_{0}, \theta_{1}}$.

Claim 2 For each even-length $s \in 2^{<\mathbb{N}}$ with $\exists x \in T_{i}(x(|x|-1) \subseteq s), s$ is in $\bigcup_{x \in T_{i}} W_{x}^{i}$ if and only if player I wins $\eta_{i}(f)$ at $s$.

Proof of Claim 2 The "if" part is clear. For the converse, by $\Sigma_{1}^{1}$ (or $\Pi_{1}^{1}$ ) transfinite induction along $T_{i}$, we prove $\forall x \in T_{i}\left(\forall s \in W_{x}^{i}\right.$ (player I wins $\eta_{i}(f)$ at $\left.s\right)$ ). By 3 and 4 of Remark 4.4, "player I wins $\eta_{i}(f)$ at $s$ " is equivalent to a $\Sigma_{1}^{1}$ formula or a $\Pi_{1}^{1}$ formula and, if $i=0$, the underlined part is equivalent to a $\Sigma_{1}^{1}$ formula by $\Sigma_{1}^{1}$ axiom of choice. Assume that, for any $y \prec_{i} x$, for any $t \in W_{y}^{i}$, player I wins $\eta_{i}(f)$ at $t$. Since $\eta_{i}(f)$ is $\Pi_{2}^{0}$ or $\Sigma_{2}^{0}$, it is determinate in ATR $\mathrm{R}_{0}$. Suppose, for contradiction, that player II has a winning strategy $\nu$ in $\eta_{i}(s * f)$ for some $s \in W_{x}^{i}$. Let $v$ be a winning strategy for player I in $\eta_{i}(s * f) \vee \exists n(s * f)[n] \in W_{\prec{ }_{i} x}^{i}$. Then there exists $n$ such that $s *(v \otimes \nu)[n] \in W_{\prec_{i} x}$, for otherwise $\eta_{i}(s *(v \otimes \nu))$ would hold and player II would lose $\eta_{i}(s * f)$. Take such least $n$. By inductive hypothesis, player I has a winning strategy $v^{\prime}$ in $\eta_{i}(s *(v \otimes \nu)[n] * f)$. Then define a new strategy $\rho$ for player I in $\eta_{i}(s * f)$ by

$$
\rho(u)= \begin{cases}v^{\prime}(u \ominus n) & \text { if }(v \otimes \nu)[n] \subseteq u, \\ v(u) & \text { otherwise. }\end{cases}
$$

Then $(\rho \otimes \nu)[n]=(v \otimes \nu)[n]$ and so $\eta_{i}(s *(\rho \otimes \nu))$ holds, which contradicts the assumption that $\nu$ is a winning strategy for player II in $\eta_{i}(s * f)$.

Then, define a game $\varphi^{*}(f) \equiv \exists n\left(f[n] \in \bigcup_{x \in T_{0}} W_{x}^{0} \cup \bigcup_{y \in T_{1}} W_{y}^{1}\right)$. We prove the following claim, which completes the proof of this theorem.

Claim 3 The player who wins $\varphi^{*}(f)$ also wins $\varphi(f)$.

Proof of Claim 3 First, assume that player I has a winning strategy $\sigma^{*}$ in $\varphi^{*}(f)$. We prove that player I has a winning strategy in the game $\varphi_{0}(f) \equiv \exists n\left(f[n] \in \bigcup_{x \in T_{0}} W_{x}^{0} \cup \bigcup_{y \in T_{1}} W_{y}^{1}\right) \wedge(\exists n(f[n] \in$ $\left.\bigcup_{y \in T_{1}} W_{y}^{1}\right) \rightarrow \eta_{1}(f)$ ), which is determined in $\Pi_{1}^{1}-\mathrm{TR}_{0}$. Suppose, for contradiction, that player II has a winning strategy $\nu^{\prime}$ in $\varphi_{0}(f)$. Then there exists $n$ with $\left(\sigma^{*} \otimes \nu^{\prime}\right)[n] \in \bigcup_{y \in T_{1}} W_{y}^{1}$, for otherwise $\left(\sigma^{*} \otimes \nu^{\prime}\right)[m] \in \bigcup_{x \in T_{0}} W_{x}^{0}$ would hold for some $m$ and player II would lose $\varphi_{0}(f)$. Take the least such $n$. By Claim 2 for $i=1$, player I has a winning strategy $v^{\prime}$ in $\eta_{1}\left(\left(\sigma^{*} \otimes \nu^{\prime}\right)[n] * f\right)$. Then we define a new strategy $\rho^{\prime}$ for player $\mathrm{I}$ in $\varphi_{0}(f)$ as follows:

$$
\rho^{\prime}(u)= \begin{cases}v^{\prime}(u \ominus n) & \text { if }\left(\sigma^{*} \otimes \nu^{\prime}\right)[n] \subseteq u, \\ \sigma^{*}(u) & \text { otherwise }\end{cases}
$$

Then $\left(\rho^{\prime} \otimes \nu^{\prime}\right)[n]=\left(\sigma^{*} \otimes \nu^{\prime}\right)[n] \in \bigcup_{y \in T_{1}} W_{y}^{1}$ and so $\eta_{1}\left(\rho^{\prime} \otimes \nu^{\prime}\right)$ holds, which contradicts the assumption that $\nu^{\prime}$ is a winning strategy for player II in $\varphi_{0}(f)$. By $\Sigma_{2}^{0}$-Det ${ }^{*}$, player I has a winning strategy $\sigma^{* *}$ in $\varphi_{0}(f)$. 
By 3 of Remark 4.4 and Lemma 4.3, we have a sequence $\left\langle\sigma_{s}: s \in \bigcup_{x \in T_{0}} W_{x}\right\rangle$ of winning strategies for player I in $\eta_{0}(s * f)$. Then define $\sigma$ by

$$
\sigma(s)= \begin{cases}\sigma_{t}(s \ominus|t|) & \text { if } t \text { is the } \subseteq \text {-least initial segment of } s \text { with } t \in \bigcup_{x \in T_{0}} W_{x}^{0}, \\ \sigma^{* *}(s) & \text { if } \forall t \subseteq s\left(t \notin \bigcup_{x \in T_{0}} W_{x}^{0}\right) .\end{cases}
$$

We can see that $\sigma$ is a winning strategy for player I in $\varphi(f)$ as follows.

Since $\sigma^{* *}$ is a winning strategy for player I in $\varphi_{0}(f)$, he can reach, following $\sigma, f[k] \in\left(\bigcup_{x \in T_{0}} W_{x}^{0} \cup\right.$ $\left.\bigcup_{y \in T_{1}} W_{y}^{1}\right)$. Take the least such $k$.

If $f[k] \in \bigcup_{x \in T_{0}} W_{x}^{0}$, then $f \ominus k$ is a resulting play of $\sigma_{f[k]}$, and so $\eta_{0}(f)$ holds. By Claim 1 and the fact $f[k] \in \bigcup_{x \in T_{0}} W_{x}^{0}, f$ also satisfies $\forall n \exists m>n \theta_{0}(f[m])$. Therefore player I wins $\varphi(f)$.

If $f[k] \in \bigcup_{y \in T_{1}} W_{y}^{1}$, then $f$ is a resulting play of $\sigma^{* *}$, and so $\eta_{1}(f)$ holds. Again by Claim 1 and the fact $f[k] \in \bigcup_{y \in T_{1}} W_{y}^{1}, f$ also satisfies $\forall n \exists m>n \theta_{1}(f[m])$. Therefore player I wins $\varphi(f)$.

Next assume that player II has a winning strategy $\tau^{*}$ in the game $\varphi^{*}(f)$. Define $\widetilde{W}^{0}$ and $\widetilde{W}^{1}$ by

$$
\widetilde{W}^{i}=\left\{s \in 2^{<\mathbb{N}}:(|s| \text { is even }) \wedge \exists x \in T_{i}(x(|x|-1) \subseteq s) \wedge s \notin \bigcup_{y \in T_{i}} W_{y}^{i}\right\}
$$

For each resulting play $f$ of $\tau^{*}$, take the least even $n \in \mathbb{N}$ and $x \in T_{0} \cup T_{1}$ with $x(|x|-1) \subseteq f[n]$. By the assumption for $\tau^{*}, f[n] \in \widetilde{W}^{0} \cup \widetilde{W}^{1}$. Note that, player II wins $\eta_{0}(f)$ at each $s \in \widetilde{W}^{0}$ and $\eta_{1}(f)$ at each $s \in \widetilde{W}^{1}$ by $\Sigma_{2}^{0}$-Det*, which is provable in ATR . Similarly to the above case, we can show that player II has a winning strategy $\tau^{* *}$ in $\neg\left(\exists n\left(f[n] \in \widetilde{W}^{0} \cup \widetilde{W}^{1}\right) \wedge\left(\exists n\left(f[n] \in \widetilde{W}^{0}\right) \rightarrow \neg \eta_{0}(f)\right)\right)$. Let $\left\langle\tau_{s}: s \in \widetilde{W}^{1}\right\rangle$ be a sequence of winning strategies for player II in $\eta_{1}(s * f)$ yielded by 1 of Remark 4.4 and Lemma 4.3. Then strategy $\tau$ for player II defined by

$$
\tau(s)= \begin{cases}\tau_{t}(s \ominus|t|) & \text { if } t \text { is the } \subseteq \text {-least initial segment of } s \text { with } t \in \widetilde{W}^{1}, \\ \tau^{* *}(s) & \text { if } \forall t \subseteq s\left(t \notin \widetilde{W}^{1}\right) .\end{cases}
$$

is a winning strategy for player II in $\varphi(f)$.

Corollary 6.6 RCA $\mathrm{RC}_{0}^{*} \vdash \operatorname{Sep}\left(\Delta_{2}^{0}, \Sigma_{2}^{0}\right)$-Det* $\leftrightarrow \Delta_{2}^{0}$-Det $\leftrightarrow \Pi_{1}^{1}-\mathrm{TR}_{0}$.

Proof. The implication from $\operatorname{Sep}\left(\Delta_{2}^{0}, \Sigma_{2}^{0}\right)$-Det* to $\Delta_{2}^{0}$-Det is proved in Theorem 6.3. The implication from $\Pi_{1}^{1}$ transfinite recursion to $\operatorname{Sep}\left(\Delta_{2}^{0}, \Sigma_{2}^{0}\right)$-Det* is proved in Theorem 6.5. The equivalence between $\Delta_{2}^{0}$-Det and $\Pi_{1}^{1}-\mathrm{TR}_{0}$ is proved in [11].

Acknowledgements. The author would like to thank prof. Kazuyuki Tanaka who suggested Wadge's hierarchy to her; prof. Hisao Tanaka who let her notice Louveau's work of the concrete description of Wadge's classes; prof. Stephen G. Simpson and the anonymous referee who gave her invaluable comments on earlier versions of the present paper.

\section{References}

[1] C. Kuratowski, Topology, vol. 1, Academic Press (1966).

[2] A. Louveau, Some results in the Wadge hierarchy of Borel sets, Cabal Seminar 79-81 Lecture Note in Mathematics 1019, Springer Verlag (1983), pp. 28-55.

[3] M. O. MedSalem and K. Tanaka, $\Delta_{3}^{0}$-determinacy, comprehension and induction, Journal of Symbolic Logic 72 (2007), pp. 452-462.

[4] M. O. MedSalem and K. Tanaka, Weak determinacy and iterations of inductive definitions, to appear in Proc. of Computable Prospects of Infinity, World Scientific. 
[5] T. Nemoto, M. O. MedSalem and K. Tanaka, Infinite games in the Cantor space and subsystems of second order arithmetic, Mathematical Logic Quarterly 53 (2007), pp. 226-236.

[6] J. B. Paris and L. A. S. Kirby, $\Sigma_{n}$ collection in arithmetic, A. Macintyre, L. Pacholski and J. Paris (eds.), Logic Colloquium '77 Studies Logic and Foundation of Mathematics, North-Holland (1978), pp. 199-209.

[7] S. G. Simpson, $\Sigma_{1}^{1}$ and $\Pi_{1}^{1}$ transfinite induction, D. van Dalen, D. Lascar, T. J. Smiley (eds.), Logic colloquium '80 Studies Logic and Foundation of Mathematics, North-Holland (1982), pp. 239-253.

[8] S. G. Simpson, Subsystems of second order arithmetic, Springer Verlag (1999).

[9] S. G. Simpson and R. L. Smith, Factorization of polynomials and $\Sigma_{1}^{0}$ induction, Annals of Pure and Applied Logic 31 (1986), pp. 289-306.

[10] J. R. Steel, Determinateness and subsystems of analysis, Ph. D thesis, University of California, Berkeley (1975).

[11] K. Tanaka, Weak axioms of determinacy and subsystems of analysis I: $\Delta_{2}^{0}$-games, Zeitschrift für Mathematische Logik und Grundlagen der Mathematik 36 (1990), pp. 481-491.

[12] K. Tanaka, Weak axioms of determinacy and subsystems of analysis II: $\Sigma_{2}^{0}$-games, Annals of Pure and Applied Logic 52 (1991), pp. 181-193.

[13] W. W. Wadge, Reducibility and determinateness on the Baire Space, Ph.D thesis in University of California, Berkeley (1983). 